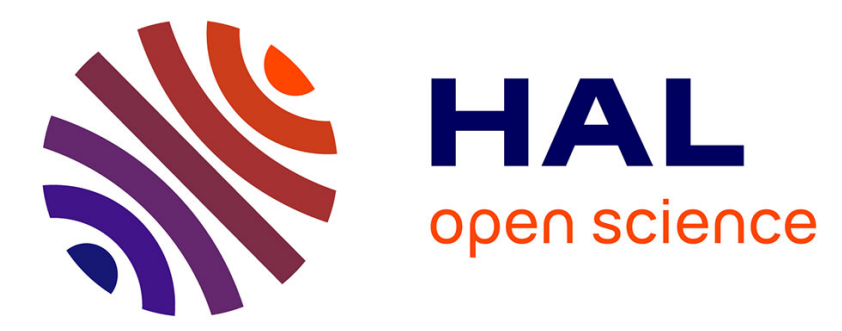

\title{
SimNCD: An agent-based formalism for the study of noncommunicable diseases
}

Rabia Aziza, Amel Borgi, Hayfa Zgaya, Benjamin Guinhouya

\section{To cite this version:}

Rabia Aziza, Amel Borgi, Hayfa Zgaya, Benjamin Guinhouya. SimNCD: An agent-based formalism for the study of noncommunicable diseases. Engineering Applications of Artificial Intelligence, 2016, 52, pp.235-247. 10.1016/j.engappai.2016.03.005 . hal-01713544

\section{HAL Id: hal-01713544 \\ https://hal.science/hal-01713544}

Submitted on 27 Feb 2018

HAL is a multi-disciplinary open access archive for the deposit and dissemination of scientific research documents, whether they are published or not. The documents may come from teaching and research institutions in France or abroad, or from public or private research centers.
L'archive ouverte pluridisciplinaire HAL, est destinée au dépôt et à la diffusion de documents scientifiques de niveau recherche, publiés ou non, émanant des établissements d'enseignement et de recherche français ou étrangers, des laboratoires publics ou privés. 


\title{
SimNCD: An agent-based formalism for the study of noncommunicable diseases
}

\author{
Rabia Aziza ${ }^{1}$, Amel Borgi ${ }^{1}$, Hayfa Zgaya $^{2}$ and Benjamin Guinhouya ${ }^{2}$ \\ ${ }^{1}$ LIPAH research laboratory, Université de Tunis El Manar, Rommana 1068, Tunis, Tunisia \\ rabia.aziza@gmail.com, amel.borgi@insat.rnu.tn \\ ${ }^{2}$ EA 2994, Public Health: Epidemiology and Healthcare Quality, University Lille, \\ 42 rue Ambroise Paré, 59120 - Loos, Lille, France \\ hayfa.zgaya@univ-lille2.fr benjamin.guinhouya@univ-lille2.fr
}

\begin{abstract}
Noncommunicable diseases (NCDs) are multifactorial chronic illnesses that cause longterm morbidities in a person's life. Their nonlinear and complex dynamics make them difficult to control and predict. Hence, it is crucial for public health researchers and practitioners to understand and model them. This paper proposes a generic interaction-oriented agent-based modeling for NCDs, called SimNCD. It models individuals living within a social network and daily engaging in activities from the physical environment. The practice of these activities stirs the individual's health vulnerability to cure, acquire or maintain NCDs. This modeling can serve as a tool in public health policies for the study of NCDs where the individual's behavior greatly influences the factors predisposing to that disease. We also propose a specified version of SimNCD for modeling childhood obesity, called SimNCD ${ }^{\mathrm{ChO}}$. More precisely, the latter models the complex relationships between children's physical activity and the development of their corpulence during their growth.
\end{abstract}

Keywords. Agent-based modeling, interaction-oriented formalism, simulation, noncommunicable diseases.

\section{Introduction}

Noncommunicable diseases (NCDs) are multifactorial illnesses that cause long-term morbidities in a person's life, e.g. bone health, obesity, cardiovascular disease, and mental disorders. Their nonlinear and complex dynamics make them difficult to control and predict, and therefore very costly for public health. Hence, it is crucial for public health researchers and practitioners to understand such complex dynamics and be able to model them. This work aims to model a generic interaction-oriented (Kubera et al., 2011) Agent-Based Model (ABM) (Wooldridge, 2009), named SimNCD, that can be adapted and implemented for the study of specific diseases. SimNCD proposes a formalism of an abstract ABM. It models individuals living in a social and physical environment that they perceive and interact with. Their behavior is mainly based on practicing activities available in their physical environment, such as performing sports or going to work. Such practice influences the individuals, and the accumulated influences over time constitute the development of the individuals' health. Such system can serve as a tool in public health policies for the study of NCDs where the individual's behavior greatly influences the factors predisposing to that disease. The said simulation tool would be useful to 
predict and explain the effects of interventions on individuals' health. In this paper, we focus on the behavioral and interactive aspect of the proposed ABM. The modeling of the cognitive processes and mental attitudes (Bagdasaryan, 2011) Will be presented in future work as an extension of SimNCD. As proof of concept, we use SimNCD in the context of childhood obesity, and we propose $\mathrm{SimNCD}^{\mathrm{ChO}}$. The latter is a system that helps simulate the complex relationships between children's physical activity (PA) and the development of their corpulence. This paper focuses on modeling the effect of PA opportunities provided by the physical environment, on the children's PA and corpulence variations. We implement $\mathrm{SimNCD}^{\mathrm{ChO}}$ and carry experimentations for the validation of the proposed modeling. To that end, we reproduce empirical data from actual field studies. Then we use the simulator to predict insights about the development of children's corpulence in different cases. This paper is structured as follows. In Section 2, we describe the epidemiological background of our work, as well as the agent-based paradigm and its usefulness in such a context. In Section 3, we provide the formal description of the proposed generic ABM, SimNCD, based on an interactionoriented agent-based approach (Kubera et al., 2011). Then, we specify this modeling for the study of childhood obesity. Section 4 describes the experimentation for both the validation of the proposed modeling and the prediction of children's corpulence in different scenarios. Finally, we conclude the paper with a glance on future work in Section 5.

\section{Background}

\subsection{Noncommunicable diseases and their risk factors}

NCDs represent a primary concern and a great burden on today's public health because of their devastating human, social and economic consequences. Nowadays, they represent the most lethal health problems around the world (WHO, 2014), and their mortality rates are estimated to escalate from 38 million in 2012 to reach 52 million by 2030. In order to decrease the damage of NCDs, public health policy-makers invest in health systems and apply promotion programs that mainly target individuals at high risk. Such programs are implemented in order to efficiently treat and cure the diseases by guiding individuals' lifestyle behaviors. They rely on awareness of the gravity of these global killers, investments, and also the comprehension of the mechanisms that lead to these diseases and maintain them (WHO, 2014). An abundant number of studies aim to understand the multifactorial liaisons that cause NCDs, such as interventions (Khan et al., 2014; Li et al., 2014). A public health intervention is a set of actions that involves a group of people and intends to make changes to prevent or treat a disease (Aziza et al., 2014; Guinhouya, 2012). In fact, the causes of NCDs range from environmental elements to personal ones. These elements are usually referred to as risk factors. NCDs share common risk factors that expose the individuals to vulnerable health conditions and make them more susceptible to diseases. These factors are dynamic and multidimensional. They stem from different aspects of the individual's life, such as the social entourage, e.g. peer influence, the physical 
environment, e.g. neighborhood security level, the person's biological factors, e.g. age and gender, and his/her behavior, e.g. tobacco use and PA. Some of these factors represent behavioral risks, like the unhealthy diet and tobacco use, while others are considered as metabolic risks, such as the raised blood glucose and obesity (WHO, 2014).

In fact, all NCDs are multifactorial. They not only share risk factors, they can also cluster and coexist in individuals causing greater health, financial, and social damages. Such multifactorial etiology renders NCDs even more difficult to understand and predict. In fact, understanding the risk factors helps identify ways to prevent or control NCDs, which is not an easy task. Indeed, the liaisons and the functioning of these risk factors are still a matter of debate. Moreover, the factors can cluster and interact, often multiplicatively. They influence each other, usually in a nonlinear manner, and raise the risk of NCDs. They could be viewed as a large graph with complex interactions and feedback loops between them. The diseases appear to be an emerging result of multiple small elevations of several risk factors (behavioral and/or metabolic) rather than caused by one specific factor (Dobe, 2012).

\subsection{Analytical approach in public health}

Traditional models are often used in the study of NCDs. They rely on analytical analysis, i.e. differential equations and stochastic processes, and statistical tools, e.g. surveys. Yet these models are reductionist. They trim the complexity of the system and focus on the data that are separately harbored by the elements, rather than the relationships and dynamics between these elements. Therefore, they fail to express the nonlinear complexity of such context (Nicolet, 2010). Hence, an appropriate modeling for NCDs should go beyond the traditional analysis in order to propose a realistic view on the system's dynamics. Such modeling should be able to be adapted to the required complexity of this field; it needs to include not only the individuals' inner states, i.e. their behaviors and health factors, but also the factors of their social and physical environments. In such context of study, the micro behaviors and intra-personal elements lead to the development of the NCDs in the population. To sum up, we aim to focus on modeling the complex and nonlinear relationships between elements at different levels and dimensions; e.g. between factors, individuals, and between an individual and the environment. Such multi-level complexity invites us to model an ABM.

\subsection{Agent-based paradigm in public health}

ABMs model agents living within their physical environments and interacting with each other. These agents are dynamic entities that move freely and independently from each other and from the physical environment. They also evolve based on a set of rules that can differ from one agent to another. Therefore, their behaviors can be heterogeneous with nonlinear dynamics. Agents can be reactive or cognitive. In the first category, reactive agents make the same decision when facing the same conditions. Their behavior is deterministic and very predictable. In the second category, cognitive agents rely on human-like reasoning mechanisms to guide their actions. They are built on an internal 
state that can be expressed via beliefs, preferences, goals, emotions, etc. e.g. (Behzadi and Alesheikh, 2013; Caballero et al., 2011). These agents deliberate over the available choices in order to choose the best decision from their point of view (Bagdasaryan, 2011; Bonabeau, 2002; Wooldridge, 2009). Besides that, just like in real life, an individual does not necessarily see the entire system, but rather keeps a perception of things around him. This perception is subjective and can be incorrect or incomplete. The agent-based paradigm allows modeling dynamics that are determined by individual actions and inter-agent interactions. The behavior of the system will emerge; it is not modeled directly. A simple and comprehensible ABM makes it easier to interpret the results, especially in the case of emerging phenomena or behaviors. It is therefore important to find a balance between modeling all identified factors and keeping it simple yet explanatory (Kantamneni et al., 2015; Kubera et al., 2011). Applied to public health, this paradigm can predict the effectiveness of health promoting programs, and anticipate their economical, social and personal effects. It can also improve our understanding of the complex dynamics in this area, and broaden the spectrum of policies, studies, and questions being raised. The literature proposes many ABMs in public health. In the study of communicable diseases, for instance, we cite the simulation of the spread of cholera (Crooks and Hailegiorgis, 2014), the diffusion of infectious diseases (Wasik et al., 2014), the study of the influence of social connections on transmitted infectious diseases (Reiner et al., 2014), and the modeling of cellular interactions (Pogson et al., 2006; Segovia-Juarez et al., 2004). As for the study of NCDs, we cite the ABM of the influence of the social dynamics on the individuals' adiposity and/or their physical activity behaviors (Hammond and Ornstein, 2014; Shoham et al., 2012; Zhang et al., 2015), the simulation of the impact of the walking school bus on children's active travel to school (Yang et al., 2014), the study of the disparities in the diet behavior within a population with racial differences (Orr et al., 2014), the effects of peer influence and friendships on the smoking prevalence (Schaefer et al., 2013), and the environmental effects on the walking behaviors of individuals (Yang et al., 2011).

Besides that, formal ABMs were also proposed in public health. Among other examples in the case of infectious diseases, the literature proposes a formal modeling for defining complex agent networks (Mei et al., 2015), a spatio-temporal specification language, EpiSpec, for describing the spread of contagious diseases (a probabilistic framework) (Hussain et al., 2014), and a formal framework for the study of HIV/AIDS, FABS-AIDS (Siddiqa and Niazi, 2013). In the case of NCDs, we cite the stochastic ABM for network dynamics, SIENA (Snijders et al., 2010). It models a network that consists of ties between agents that change over time, and it allows representing the stochastic dependence between the creation and termination of these ties. The dynamics governing the interagent relationships are based on observed longitudinal and/or cross-sectional data, and they are described via stochastic Markov processes, i.e. processes where the prediction of the future state of the network is based on its current state, not its history. SIENA was applied in several studies, e.g.

(Schaefer et al., 2013; Shoham et al., 2012; Zhang et al., 2015).

Unfortunately, despite its increase during the last decade, the use of ABMs for modeling NCDs is still 
considered scarce and relatively new. In this subject, Nianogo and Arah state that: "Although increasingly used to study NCDs, ABMs remains underutilized and, where used, is sub optimally reported in public health studies" (Nianogo and Arah, 2015). Withal, these models usually focus on only one aspect of the complex dynamics of NCDs, e.g. the social influence (Hammond and Ornstein, 2014), the physical environment (Yang et al., 2011), or the economic aspect (Auchincloss et al., 2011). Moreover, they explain the hypothesis and the different elements they model. Yet most of them do not describe a clear manner to extend these models in order to include other risk factors. For example, most of them do not include the spatial dimension (i.e. physical environment), which is an important component that influences the individual's behaviors (WHO, 2014).

In addition, most proposed $\mathrm{ABMs}$ fail to offer a detailed formal conceptual description that could help implement and use them. In fact, the model description is often textual, and it is sometimes accompanied by equations or diagrams (Nianogo and Arah, 2015). While these diagrams help understand the functioning of the ABM and the equations do support and clarify the hypothesis of the system, we believe a transparent formalized modeling is necessary if the latter is meant to be actually used by public health practitioners. Besides that, NCDs often share common risk factors. Therefore, it would be advantageous in some cases to reuse the modeling of some factors that were initially modeled for one NCD, in the modeling of another NCD. This kind of recycling reduces the resources for designing ABMs. It would also draw out the similarities in the hypothesis and points of view of different public health researchers, hence further stimulating discussions on the matter. In such case, it would be helpful if these models shared the basic formal description.

The formal ABMs proposed for public health, such as EpiSpec (Hussain et al., 2014) and SIENA (Snijders et al., 2010), model diseases as stochastic social networks. They describe the dynamics of the system stochastically. Although such models were proven useful to public health, we believe they limit the way we describe the individual's behaviors and his/her risk factors as they allow only stochastic dynamics. They also focus mostly on the social facet of the studied context. In our opinion, a good formal modeling for NCDs should allow representing different kinds of dynamics, e.g. based on rules, algorithms, or probabilities. It should also be unbiased towards the aspects of a person's life that the researcher wants to model. In other words, the choice of modeling the individuals as agents that have a social, cognitive, or physical identity should be left for the designer to make. For instance, in the sub-section 3.4, we study the effects of the physical environment on the individuals' health, without necessarily diving into the dynamics of their social relationships.

In this work, we propose a modeling that aims to offer such a formal description of an ABM in the context of NCDs, called SimNCD. This modeling is generic because it is not related to a specific disease. Instead, it offers an abstract formal modeling that includes the basic necessary elements to model the individuals' lives and the development of their health, namely the contextual elements, i.e. physical and social environments, and an inner status revolving around health risk factors. SimNCD can be oriented by public health researchers for the investigation of specific hypothesis or 
interventions in their field.

\subsection{Interaction-oriented agent-based modeling}

The literature proposes two ABM approaches: the task-centric modeling (also known as contentcentric) and the interaction-oriented modeling. The task-centric approach concentrates on what tasks the agent needs to accomplish and what data it should have. The agent's behaviors are modeled via algorithms, which can lead to complicated models with quite heavy information to manage, and the interactions are implicitly modeled within the tasks based on communication protocols. The modeling of the inter-agent communications tends to complicate the behaviors even more. On the other hand, the interaction-oriented approach focuses mainly on the interactions. It designs an interaction as an explicit and autonomous entity that represents a behavior of an agent. It is modeled independently from the agent and vice-versa, and it can involve communicating with other agents or not (Kubera et al., 2011). This facilitates the modeling and allows the designer to focus on the influences between the elements of the system: each action or behavior of an agent is designed as an interaction. In this paper, we opt for an interaction-oriented approach, and we use IODA (Interaction Oriented Design of Agent simulations) (Kubera et al., 2011). IODA focuses on the influences and the interactions between the entities of an $\mathrm{ABM}$. According to our review of the literature, it is so far the only approach that models interactions as explicit and autonomous entities on which the agent's behavior is based, and not via interaction protocols and communications that are linked to the behaviors (and implicitly modeled in them). IODA offers a formalism for describing ABMs, a topdown incremental methodology, and a Model-View-Controller implementation framework JEDI (Java Environment for the Design of agent Interactions). It has two main advantages. The first is that this approach is cross-sectional; all concepts described in the modeling phase are implementable as code snippets in the simulation phase. The second main advantage is that the interactions are designed independently of the agents, and vice-versa. Once all interactions are modeled separately, they are assigned to the agents via an interaction matrix (Kubera et al., 2011).

\section{Model design}

We begin by presenting a multi-level architecture of our ABM. Then, we present a specification of this modeling for the study of childhood obesity as a proof of concept.

\subsection{Multi-level architecture of SimNCD}

First, we would like to note the difference between the environment, the physical environment, and the social environment in our ABM. In any ABM, all agents live within a technical structure that surrounds them: the environment. In our case, we model it as a 2D grid where all the entities coexist. As for the physical (or social) environment, it represents the health risk factors that stem from the physical (or social) entourage of the individual, e.g. the safety of the neighborhood or the mother's 
smocking behavior. In fact, as depicted in Figure 1, our systems models individuals living in a social and physical environment that they perceive and interact with, while developing their inner states. The social network of a person is the set of other individuals that he/she knows and interacts with. The physical environment is modeled as a set of activities that the individuals can practice, e.g. swim, do puzzles, go to school, and play in the garden. The intra-individual level includes the individual's behaviors, cognitive abilities, perceptions, and all the risk factors considered in the studied context. These factors include the environmental factors (social or physical) that the individual is aware of due to his/her perception mechanisms. We believe such architecture allows a gradual integration of the various factors that regulate the relations between the individual's behaviors and the studied NCD. In SimNCD, we have activities and individuals. We describe them as agents by defining their following elements: the percepts, the actions (i.e. behaviors, also referred to as interactions in IODA), the goals, and the environment (Padgham and Winikoff, 2005; Russell and Norvig, 2013). We start by modeling the activity agents, and then we model the individual agents.
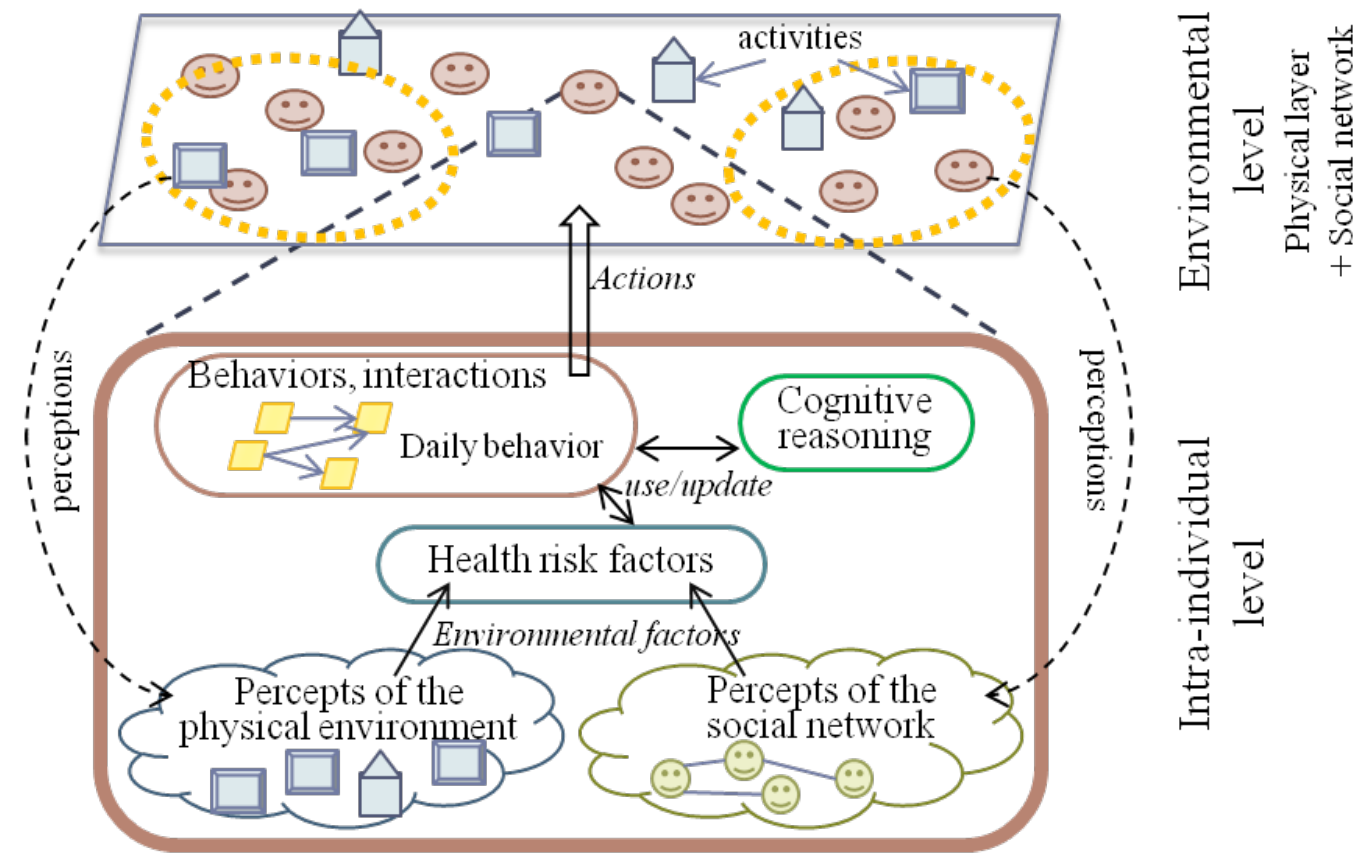

Figure 1. Agent-based architecture of SimNCD (Aziza et al., 2014).

\subsection{Modeling the activities}

IODA considers all entities in the system as agents stemming from at least one agent family. An agent family is an abstract specification of an agent (Kubera et al., 2011). It is equivalent to Classes in Object Oriented modeling. Thus, the activities are described via an agent family that we note: $\mathrm{F}^{\text {Activity }}$. Instances of $\mathrm{F}^{\text {Activity }}$ are simple reactive agents. They constitute the physical environment, and they represent the activities that are available for the individuals to practice. Thus, $\mathrm{F}^{\text {Activity }}$ describes characteristics. These latter depict the features of the physical environment that are relevant to the studied context, e.g. in the study of the consummation of healthy foods in a population (as in (Zhang et al., 2014)) we can create the activity 'buying foods from a store', and this activity specifies if it allows 
purchasing fresh fruits and vegetables. An activity can also describe the constraints that specify the restrictions on the individual's participation in the activity, e.g. an activity could restrict its access to only a specific age range. We do not restrict the way to describe the characteristics and the constraints because they depend greatly on the application of SimNCD (the specific studied disease).

Moreover, instances of $\mathrm{F}^{\text {Activity }}$ do not reason nor initiate any behaviors. Nevertheless, we allow them to perceive the individuals who practice them. This means that an activity has a perception mechanism. However, this mechanism is not triggered by the activity itself, rather the individuals who engage or disengage in it, i.e. practice it. Therefore, the behaviors that allow an activity to (un)perceive an individual will be detailed in the description of the interactions initiated by the individuals.

\subsection{Modeling the individuals}

The second type of entities in our ABM concerns the individuals who represent the social network of SimNCD. We describe them using an agent family that we note $\mathrm{F}^{\text {Individual }}$. We model their daily behavior, and then we formalize this behavior via interactions. Finally, since our context revolves around NCDs, we model the individuals' health factors, and we explain how the factors and perceptions evolve.

\subsubsection{Modeling the individual's daily behavior}

Each individual engages in some of the available activities (instances of $\mathrm{F}^{\text {Activity }}$ ) every day, which defines his/her daily behavior. The activities can be of various kinds, e.g. leisure, outdoor, and educational activities, and their practice can have different effects on the individual's health. And while practicing one, the individual may interact with other individuals, alter the physical environment and modify his/her inner status.

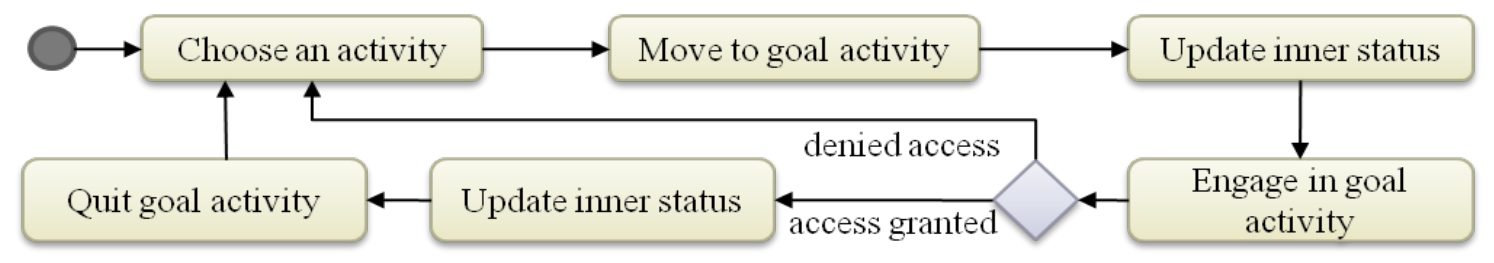

Figure 2. Activity diagram of an individual agent in the proposed agent-based model.

We model an individual's daily behavior as illustrated in Figure 2. An individual starts by choosing the activity to practice. This choice can be randomized or sequential from a set of perceived activities. It can rely on more complex reasoning mechanisms, in which case the choice will be cognitive. In SimNCD, engaging in activities is the only goal of an individual agent in our model. Each time an individual chooses an activity, we consider that activity to be the 'goal activity'. Afterwards, the individual moves towards its goal in order to attempt to engage in it. This movement can be considered as an activity itself, e.g. taking the bus, walking, or riding a bike. It is not the chosen goal activity, but it might involve an effort from the individual. In such case, we take this effort into consideration by updating the individual's inner status based on the characteristics of the movement. 
The way to model this movement is left to the specification of the generic modeling that we propose; it could be, for instance, modeled as an activity itself. Then, when reaching the desired goal activity and attempting to engage in it, the access can be denied to the individual in case he/she doesn't meet the specific requirements described in the activity's constraints, e.g. having the appropriate age, and he/she has to choose another goal activity. Otherwise, the access is granted and the individual performs the activity. Before concluding the practice and quitting the current goal activity, the individual updates his/her inner status. The same process is applied to all the practiced activities during each day of the development of the individual.

An agent can only interact with the agents it perceives (Wooldridge, 2009). We allow the individuals

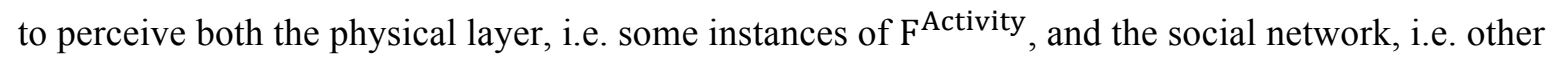
instances of $\mathrm{F}^{\text {Individual }}$. Basically, the individuals need to perceive some activities in order to be able to 'live' according to the process depicted in Figure 2. The act of (un)perceiving an activity or another individual should be modeled as a behavior, i.e. interaction. We believe the detailed specification of the mechanisms of perception of the children is highly dependent on the studied domain. Therefore, in sub-sections 3.3.2 and 3.3.3, we describe the main concepts/elements that allow modeling such mechanisms. And these concepts need to be specified in details when studying specific NCDs.

\subsubsection{Interactions}

All agents in our ABM behave and interact based on interactions. These latter have source and target agent families. The source is the agent family that initiates the interaction, and the target is any agent family involved/affected by the source. We gather the interactions that occur in SimNCD and that reflect the individual's behavioral model (Figure 2) in a set I. Since they are all initiated by the individuals, $\mathrm{F}^{\text {Activity }}$ can be considered a passive agent family, and the source agent family is always $\mathrm{F}^{\text {Individual }}$. We translate the individual's behavior into the following interactions:

- I ChooseGoalActivity allows the agent to choose the next goal activity to pursue. It involves only the agent itself, and the goal activity needs to be perceived.

- I $^{\text {Move }}$ moves the individual across the environment and towards the goal activity. This interaction also involves only the agent itself.

- I Engage allows the individual to access the activity by interacting with it. It involves the individual as well as the goal activity. Besides that, any access constraints set by the activity need to be checked at this step. If the access is granted, we alter the activity's perception by registering the individual as a current participant.

- I ${ }^{\text {Quit }}$ is executed once the practice of the current goal activity is finished. It occurs between the individual and the goal activity. It alters the activity's perception by removing the individual from the activity's current participants.

We note that $\mathrm{I}^{\text {Engage }}$ and $\mathrm{I}^{\text {Quit }}$ are responsible for allowing the activities to (un)perceive the 
individuals who practice them. Thus, $\mathrm{I}^{\text {Engage }}$ and $\mathrm{I}^{\text {Quit }}$ are the actions that maintain the perception in $\mathrm{F}^{\text {Activity }}$. Besides that, we add another interaction that applies the updates to the individual's inner status, e.g. increase the person's blood pressure. This case concerns the behavior 'Update inner status' that happens twice in the individual's behavioral process (Figure 2): once to apply the effects of moving towards the goal activity, and another to apply the effects of practicing the goal activity. In fact, the updates are micro effects performed on the agent's inner state and its social or physical environment. In other words, the updates can translate: an act of perception of the physical environment (e.g. perceiving the current neighborhood as insecure), a perception of the social network (e.g. identifying the friends among the participants of the same activity), an action on either the physical or social environments (e.g. bullying a peer), or a development in the individual's own inner state (e.g. altering the level of energy intake). With time, the development of the individuals emerges from the combination of all these micro updates. Contrary to the other interactions, the update is abstract because we still do not know what exactly occurs in the individual's health risk factors; such specification is made when using SimNCD in the study of a particular NCD.

Let us consider $\psi^{\mathrm{F}^{\text {Individual }}}$ as an abstract update, see expression (1). An update can occur between more than one individual, $\psi_{\mathrm{F}^{\text {Individual }}}^{\mathrm{F}^{\text {Indididual }}}$, an individual and an activity, $\psi_{\mathrm{F}^{\text {Activity }}}^{\mathrm{F}^{\text {Individual }}}$, or it can involve only the individual himself/herself, $\psi_{\varnothing}^{\mathrm{F}^{\text {Individual }}}$. All these categories are updates applied by instances of $\mathrm{F}^{\text {Individual }}$. And any declared update must ascend from one of these categories. The total set of interactions I becomes as displayed in expression (2).

$\psi^{\mathrm{F}^{\text {Individual }}}=\left\{\psi_{\emptyset}^{\mathrm{F}^{\text {Individual }}}, \Psi_{\mathrm{F}^{\text {Individual }}}^{\mathrm{F}^{\text {Individual }}, \psi_{\mathrm{F}^{\text {Activity }}}^{\mathrm{F}^{\text {Individual }}}}\right\}$

$\mathrm{I}=\left\{\mathrm{I}^{\text {ChooseGoalActivity }}, \mathrm{I}^{\text {Move }}, \mathrm{I}^{\text {Engage }}, \mathrm{I}^{\text {Quit }}\right\} \cup \psi^{\mathrm{F}^{\text {Individual }}}$

We proceed to assign the interactions with their proper agent families. We need to specify the priority between them, so that if the system faces a situation where more than one interaction could be initiated during the same simulation step, the system chooses the one with the higher priority. IODA defines priorities by giving a number to each assigned interaction; the lower the number, the higher the priority (Kubera et al., 2011). In our case, we rely on the chronological order of the interactions, as described in Figure 2, to determine their priority order. And we assign the interactions to their agent family sources and targets in the interaction matrix illustrated in Table 1. The matrix should be read following the ascending order of the priorities.

Table 1. The interactions and updates of the proposed generic ABM for the study of the relationship between individual's daily behavior and noncommunicable diseases.

\begin{tabular}{llll}
\hline $\begin{array}{l}\text { target } \\
\text { source }\end{array}$ & $\varnothing$ & F $^{\text {Individual }}$ & F $^{\text {Avtivity }}$ \\
\hline \multirow{2}{*}{$\mathbf{F}^{\text {Individual }}$} & $\left(\mathrm{I}_{\text {ChooseGoalActivity }}, 0\right)^{\mathrm{a}}$ & - & $\left(\mathrm{I}_{\text {Engage }}, 2\right)^{\mathrm{a}}$ \\
& $\left(\mathrm{I}_{\text {Move }}, 1\right)^{\mathrm{a}}$ & $\left(\mathrm{I}_{\text {Quit }}, \max (\mathrm{x})+1\right)^{\mathrm{a}}$ \\
\hline
\end{tabular}




\begin{tabular}{|c|c|c|c|}
\hline & $\left(\psi_{\emptyset}^{\mathrm{F}^{\mathrm{Individual}}}, \mathrm{x}\right)^{\mathrm{b}, \mathrm{c}}$ & $\left(\psi_{\mathrm{F}^{\text {Individual }}}^{\mathrm{F}^{\text {Individual }}, \mathrm{x}}\right)^{\mathrm{b}, \mathrm{c}}$ & $\left(\Psi_{\mathrm{F}}^{\mathrm{F}^{\mathrm{Indinividual}}}, \mathrm{x}\right)^{\mathrm{b}, \mathrm{c}}$ \\
\hline$F^{\text {Activity }}$ & - & - & - \\
\hline
\end{tabular}

When specifying SimNCD for the study of one or more particular NCDs, we need to precisely define the updates that might occur. Each update inherits one of the three abstract updates in $\psi^{\mathrm{F}^{\text {Individual }}}$ (expression (1)) depending on the update's target agent family. By consequence, all updates are modeled as interactions and added to the interaction matrix. A particular attention should be given when attributing priorities to these updates in case some of them influence others. In general, we can suppose that the updates related to perception should be given priority because, usually, agents first perceive and then act accordingly.

\subsubsection{Modeling the individual's health}

Our modeling allows a thorough study of the development of the health according to personal and contextual dynamic factors that are predisposing directly or indirectly to NCDs. These factors influence each other, rendering them complex to decipher and understand. It is compelling to model the individual's health risk factors. In SimNCD, updating an individual's inner status amounts to updating these factors, e.g. increase the cholesterol level, alleviate the mood, and change the corpulence status. This update happens at each practice of an activity offered by the physical environment. More specifically, the evolvement of the individuals' health will be modeled in the interaction set I via the abstract updates (expression (1)).

We categorize these health risk factors in groups that mirror the different aspects of a person's life that influence (directly or indirectly) his/her health. Based on our readings, we identified the main factor groups in NCDs and we present them in Table 2.

Table 2. The main factor groups in the context of NCDs (Guinhouya et al., 2010; WHO, 2014).

\begin{tabular}{ll}
\hline Factor groups & Examples of risk factors \\
\hline Demographic & Age, gender, biological maturity. \\
Psychological & Confidence, preferences, motivation, depressive symptoms. \\
Psychosocial & Social support, encouragement, perceived quality of relationships. \\
Social & Siblings, level of parental education, parental behaviors. \\
Physical environment & Availability of bike paths, neighborhood safety, opportunity to PA. \\
Genetic & Dopaminergic sensitivity. \\
Physiological & Muscular endurance, motor skills, Body Mass Index, birth weight. \\
Behavioral & Sedentary and PA behaviors, smoking, eating behaviors. \\
\hline
\end{tabular}

Let $\mathrm{f}^{\mathrm{l}}$ be a risk factor with the label 1 as its name. A snapshot of the factors gives the needed information to interpret and evaluate the individual's health status at a given time. These factors are altered based on inter-factor influences. We model these latter using updates that we note $\psi_{\mathrm{f}^{\mathrm{ftargets}}}^{\mathrm{f} \text {, }}$, where $\mathrm{f}^{\text {targets }}$ is the set of altered factors and $\mathrm{f}^{\text {sources }}$ is the set of factors used as input to perform the 
update. Each update inherits from one of the abstract updates in the set $\psi^{\mathrm{F}^{\text {Individual }}}$ (expression (1)) depending on the agent families that need to be included in the interaction.

For instance, an individual could update his/her age. This update should inherit from the abstract update $\psi_{\varnothing}^{\mathrm{F}^{\text {Individual }}}$. An individual could also alter his/her mood (or attitude) based on the attractiveness of the place where an activity is held. This update translates a perception of the physical environment as well as a cognitive interpretation of this perception. It should inherit from $\psi_{\mathrm{F}}^{\mathrm{F}^{\text {Individual }} \text { Activ }}$. As a last example, we consider an individual who is influenced by his/her peers. Such influence means that the individual perceived those peers, and his/her inner status was affected by this perception. This could be modeled as an update that inherits from $\psi_{\mathrm{F}^{\text {Individual }}}^{\mathrm{F}^{\text {Individual }}}$ Using such modeling, we offer a generic way to specify how of the individual's perceptions and health factors evolve.

These interactions could also include decision-making mechanisms. For instance, the choice of the next activity to practice could rely on the individual's mental attitudes, e.g. preferences. In such cognitive context, the mental attitudes would be modeled as factors that not only evolve (via updates), but also influence the individual's behavior. In this paper, we focus on the basic behavioral and interactive aspect of our ABM; we do not describe the cognitive aspect of the individuals, and we model them as reactive agents. However, we note that by using the risk factors to represent cognitive attitudes, and the interactions (including the updates) to model reasoning mechanisms, SimNCD does allow modeling cognitive individuals. In fact, our current work revolves around extending SimNCD to allow the individuals to reason and make decisions based on their mental attitudes, more specifically, their preferences. This work will be published soon.

To sum up, the agents in SimNCD can be briefly described as in Table 3. The proposed formalism allows modeling inter-individual relationships as interactions (inheriting from the abstract update $\psi_{\mathrm{F}^{\mathrm{Individual}}}^{\mathrm{F} \text { Individual }}$. But, unlike EpiSpec (Hussain et al., 2014) and SIENA (Snijders et al., 2010), it also focuses on modeling the interactions between the individuals and their environment (using the update $\psi_{\mathrm{F}^{\text {Activity }}}^{\mathrm{F}^{\text {Individual }}}$ ), as well as the spontaneous development of the individual's inner state (using the update $\psi_{\varnothing}^{\mathrm{F}^{\text {Individual }}}$ ), e.g. the individual's growth depends only on the time. Besides that, SimNCD does not limit the system's development to stochastic network dynamics. Such dynamics are left to the user of the SimNCD formalism to specify according to the studied disease. In the following, we illustrate an example of specification of SimNCD with a case study about childhood obesity.

Table 3. Brief description of the agents in SimNCD based on the PAGE (Percepts, Actions, Goals, Environment) description (Russell and Norvig, 2013).

\begin{tabular}{lllll}
\hline Agent & Percepts & Actions & Goals & Environment \\
\hline Activity & $\begin{array}{l}\text { Can perceive the individuals that } \\
\text { are currently practicing this } \\
\text { activity. The individuals are }\end{array}$ & None & None & A 2D grid \\
& & & where all the \\
activities are
\end{tabular}




\begin{tabular}{|c|c|c|c|c|}
\hline & $\begin{array}{l}\text { perceived and unperceived via the } \\
\text { interactions I Engage and I }{ }^{\text {Quit }}\end{array}$ & & & $\begin{array}{l}\text { located and } \\
\text { all the }\end{array}$ \\
\hline Individual & $\begin{array}{l}\text { Can perceive a subset of the } \\
\text { available activities as well as a } \\
\text { subset of the other individuals. }\end{array}$ & $\begin{array}{l}\text { Practices activities and } \\
\text { updates his/her risk } \\
\text { factors (see Table 1). }\end{array}$ & $\begin{array}{l}\text { Practicing } \\
\text { the } \\
\text { activities }\end{array}$ & $\begin{array}{l}\text { individuals } \\
\text { live. }\end{array}$ \\
\hline
\end{tabular}

\subsection{Case study: Childhood obesity}

We use the formal description of SimNCD in order to propose a modeling for the study of the complex relationship between the PA and infantile obesity for children from 6 to 18 years old. We call this specified modeling SimNCD ${ }^{\mathrm{ChO}}$.

\subsubsection{Context of the case study}

In 2014 , the WHO reported that $11 \%$ of men and $15 \%$ of women aged 18 years and older were obese, and that in 2013, 6.3\% (equivalent to 42 million) of children under 5 years old were overweight. The latter global prevalence is estimated to rise to $11 \%$ worldwide by 2025 unless serious measures were taken to prevent it (WHO, 2014). In fact, childhood overweight and obesity is not only associated with high death rates, it is also a long-term disability that tends to persist through adulthood. This epidemic plays a key role as a mediator for several NCDs, including diabetes, musculoskeletal disorders, and anxiety. The literature proposes two main axes that fight obesity: The first is encouraging PA by using education and social marketing campaigns, along with enhancing the physical environment with better opportunities to practice PA. The second course of action is oriented towards healthy food consumption by applying the proper marketing and fiscal policies (Guinhouya, 2012; WHO, 2014). "Research is urgently needed to evaluate the effectiveness of interventions to prevent and control obesity", the WHO states (WHO, 2014). Such evaluation requires an understanding that is as deep and precise as possible of the determinants predisposing to the development of this NCD.

In this case study, we focus on modeling the PA behavior and its complex relationship with obesity for schooled children between 6 and 18 years old. In fact, PA is a complex and multidimensional behavior determined by numerous factors. Guidelines for PA have been established for children from 6 to 18 years old (WHO, 2014). These guidelines recommend a minimum level of Moderate to Vigorous PA (MVPA) in duration and/or frequency (e.g. at least 60 min three times a week (WHO, 2014)). Such recommendations aim to improve children's health and quality of life. In fact, PA takes on a particular importance in the fight against several NCDs through its nonlinear action on infantile obesity and its comorbidities, such as bone health and arterial hypertension. Nevertheless, the implementation of strategies and effective interventions requires a better understanding of the complex and nonlinear relationship between it (and related behaviors such as the sedentary behavior) and the corpulence variations during child development (Dobe, 2012; Guinhouya, 2012).

In this section, we model $\mathrm{SimNCD}^{\mathrm{ChO}}$ : a specification of $\mathrm{SimNCD}$ for childhood obesity. It aims to decipher and better understand the complex dynamics between the PA and the children's corpulence 
variations during growth. Our focus will be on modeling the effect of PA opportunities provided by the physical environment on the activity level of children. Then, we will examine the indirect effect of the resources in the physical environment on the variations in their corpulence. This work is meant to be extended later to include more risk factors. For clarity, we begin by presenting the risk factors that we consider in $\mathrm{SimNCD}{ }^{\mathrm{ChO}}$. Then we describe the interactions, including the updates and perceptions.

\subsubsection{Factors}

The literature proposes a bidirectional relationship between the children's PA and their corpulence variations. In this paper, we consider the influence of the PA behavior on the corpulence. We present the included factors and we build the influences between them.

i. Modeling the PA risk factor and related updates

We begin by modeling the child's PA; we describe this factor, noted $\mathrm{f}^{\mathrm{PA}}$, and how it evolves with time. The children's PA behavior includes several kinds of activities, such as daily activities (e.g. gardening), sports and exercise (i.e. coordinated and organized activities), and leisure activities (e.g. doing puzzles). It can be measured subjectively (e.g. with questionnaires) or objectively (e.g. via accelerometers). Besides that, it can be described using three characteristics: duration (e.g. in minutes), frequency (e.g. twice a day, three times a week), and intensity (Guinhouya, 2012). Most studies (Guinhouya, 2012) describe the latter using the linguistic terms \{sedentary, light, moderate, vigorous\}. We measure the PA risk factor using the minutes that the child spends each day in practicing MVPA. In other words, we model the daily duration of MVPA.

After describing the factor, we look into how it develops. First, we need to take into account the fact that the PA depends greatly on the child's age and gender (Guinhouya, 2012; WHO, 2014). For instance, the MVPA decreases gradually (but not uniformly nor equally between genders) from 6 to 18 years old, yet boys usually have higher MVPA than girls at all ages. Such differences are important to consider in the model because of two reasons. First, they reflect the natural course of MVPA during growth and development when all other factors are held constant. Second, it has a direct incidence on the interpretation of a child's PA level. For instance, let us consider three girls of 6, 10, and 17 years old, who are engaged in the same daily amount of MVPA $50 \mathrm{~min} /$ day. Their PA level cannot be interpreted the same way: the 6 year-old girl could be considered as inactive while the 10 year-old could be considered as normally active, and the 17 year-old could be considered as highly active. Such difference is important to consider because it is mirrored in the effects on their respective health. Thus, we declare two biological risk factors, $\mathrm{f}^{\text {Gender }}$ and $\mathrm{f}^{\text {Age }}$. The gender is a constant data. As for the age, it evolves continuously within the targeted range $[6,18]$. For that, we declare an update that manages such growth, e.g. the child's age is daily incremented by one. The age progresses with time and calls for no communication with other agents. Hence, its update is a specification of the abstract interaction $\psi_{\varnothing}^{\mathrm{F}^{\text {Individual }}}$ from expression (1), noted $\psi_{\mathrm{f}}^{\emptyset}$. 
As we said earlier, the PA depends on the child's age and gender. Let MVPA ${ }^{\alpha}$ be the minutes of MVPA of the child $\alpha \in \mathrm{F}^{\text {Child }}$, and basicMVPA( $\mathrm{f}^{\text {Age }}, \mathrm{f}^{\mathrm{Gender}}$ ) be a function that provides the amount of MVPA minutes each child would be engaged on account for the normal course of MVPA. This function relies on data from the literature where attempts were made to quantify such normal course of the children's MVPA (Guinhouya, 2012). In order to interpret PA, we use the percentage of MVPA that we call MVPA $\%$ $\alpha$ and calculate it as follows:

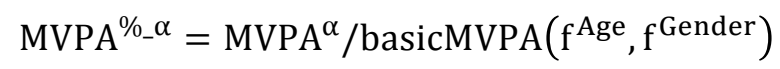

If MVPA $^{\%} \_\alpha<1$, the child $\alpha$ is considered inactive (the less MVPA ${ }^{\%} \_$, the more inactive the child would be) and this level is expected to have negative effects on his/her health. If MVPA $\%$ _ $\alpha>1$, the child is highly active and it is expected to have positive effects on his/her health. And if MVPA ${ }^{\%} \alpha=1$, then the child is normally active, and by considering all other factors stable, he/she is expected to be healthy. The latter case illustrates a child adhering to the daily recommendations of PA (i.e. 60 min/day (WHO, 2014)). With such modeling, we simplify some of the complexity of the PA behavior because MVPA ${ }^{\%} \_\alpha$ informs us of the children's level of PA independently of their age and gender, while taking into account the natural course of such a complex behavior.

Besides age and gender, the third element that influences the PA behavior in our modeling is the physical environment. For that, we need to describe the environment's characteristics that we wish to include, and the way they are perceived. Our approach consists in computing the daily MVPA that each child accumulates by engaging in the different activities offered by his/her physical environment during his/her development over the years. Thus, each activity offers a specific amount of opportunity to engage in a MVPA. It is difficult to find the exact amount of contribution of each activity to the MVPA in the literature, especially when the PA behavior depends greatly on the child's age and gender. Therefore, we draw inspiration from the work of Guinhouya (Guinhouya, 2010) and we model the opportunity to MVPA as a percentage. The latter reflects the contribution of an activity in the total MVPA of a day that is supposed ideal; during an ideal day, the sum of the opportunities to MVPA of the activities practiced by the child is $100 \%$. Such a day is considered favorable for the child's health, i.e. it allows children to meet the MVPA recommendations, and we consider it to be our reference for defining the opportunities of the activities offered by the physical environment, e.g. the activity 'play in the garden' may provide 0.3 opportunity to MVPA, while 'perform sports' may provide 0.6. Our modeling allows us to qualify the physical environment of a child by calculating the sum of the opportunities to MVPA offered by the activities practiced during the day. If such sum is less than $100 \%$, then the physical environment is considered unfavorable, if it is equal to $100 \%$, then the physical environment is favorable, and if it is more than $100 \%$, then the physical environment is as highly favorable. Such interpretation helps understand the results that the ABM produces.

The opportunity to MVPA is held by the activities. It is a characteristic expressed in percentage. This information is also perceived by the child via a risk factor that we note $\mathrm{f}^{\text {opp }}$ from the physical 
environment group of factors. In order to perceive, the agent must perform an action. Therefore, we declare an update $\psi_{\mathrm{f} \text { opp }}^{\emptyset}$ that takes care of perceiving the opportunity to MVPA. This perception will simply read the characteristic of the practiced activity, and store its value in $\mathrm{f}^{\mathrm{opp}}$. This act of perception is triggered when the child is already engaged in a goal activity. Thus, it inherits from the abstract update $\psi_{\mathrm{F}}^{\mathrm{F}^{\text {Activity }} \text {. }}$

The child practices his/her chosen goal activity and gathers an amount of MVPA depending on the activity's opportunity to MVPA, but also on how much the child profits from this opportunity. In fact, different children can have different attitudes towards the same activity: some can be considered normally motivated, and in case the physical environment is favorable, these children are expected to be active and meet the PA recommendations, e.g. $60 \mathrm{~min} /$ day of MVPA (WHO, 2014). Others can be unmotivated and exploit less than what the activity contributes to an ideal day; the lesser these children exploit the opportunities they perceive, the more inactive they tend to be. Children can also be highly motivated and exceed the PA recommendations. Let $\mathrm{f}^{\text {Attitude }}$ be the child's willingness/motivation to practice the opportunities to MVPA. Such factor is inspired from other works in the literature, such as the attitude towards active traveling to school (Yang et al., 2014), the attitude towards walking (Yang et al., 2011), and the attitude towards drinking (Garrison and Babcock, 2009). We model $\mathrm{f}^{\text {Attitude }}$ as a coefficient in [0,2]. It can be interpreted as motivated if equal to 1 , unmotivated when less, and highly motivated when more. This factor stems from the cognitive factor group. It can be influenced by other factors, e.g. the preferences, the enjoyment of practicing PA, and the peer relationships. In this modeling, however, we suppose that it is an input to our ABM. Having declared the risk factors that influence the PA in our modeling, i.e. $\mathrm{f}^{\text {Age }}, \mathrm{f}^{\text {Gender }}$, $\mathrm{f}^{\text {opp }}$, and $\mathrm{f}^{\text {Attitude }}$, we move to describe the interaction that carries out such influence. This interaction is an

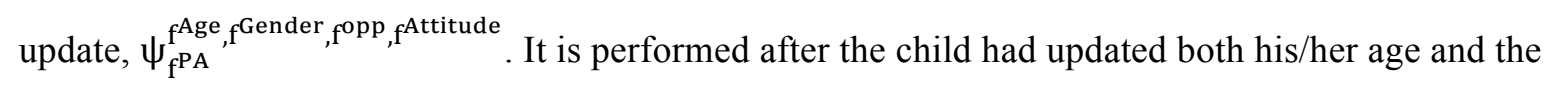
perceived opportunity to MVPA. It calculates the MVPA gathered by a child $\alpha$ after the practice of a goal activity $\beta_{\mathrm{i}}$ as depicted in (4). The daily MVPA of a child $\alpha, M \mathrm{PA}^{\alpha}$, can be calculated by summing the different $\mathrm{MVPA}_{\beta_{\mathrm{i}}}^{\alpha}$ obtained from the practice of all the activities $\beta_{\mathrm{i}}$ of that day. The factor $\mathrm{f}^{\mathrm{PA}}$ is the history of the daily MVPA of the child.

$\operatorname{MVPA}_{\beta_{\mathrm{i}}}^{\alpha}=\mathrm{f}^{\text {opp }} *$ basicMVPA $\left(\mathrm{f}^{\text {Age }}, \mathrm{f}^{\text {Gender }}\right) * \mathrm{f}^{\text {Attitude }}$

ii. Modeling the corpulence risk factor and related updates

An individual's corpulence can be characterized as \{underweight, normal, overweight, obese\} (WHO, 2007). It is a physiological factor that can be objectively measured using, among other examples, the waist circumference, ratio fat mass to fat free mass, and the BMI (Guinhouya, 2012). The latter, BMI, is defined as weight $/ h_{e i g h t}{ }^{2}\left(\mathrm{~kg} / \mathrm{m}^{2}\right)$. It is easy to calculate compared to most of the other measures, and it is the most commonly used measure in the literature. Thus, we use it as a measure of the corpulence in our model. 
The literature provides several growth references of BMI. We rely on the WHO references describing the development of the BMI of children from 5 to 18 years old, depending on their gender and age (WHO, 2007). In our model, these curves are considered as the natural growth curves of children. Let basicBMI be the BMI reference function (i.e. the natural growth of the children). It depends on the child's age, gender, and current weight status. And since, in real life, children with the same age, gender and weight status can still have different BMI, let BMI ${ }^{\alpha}$ be the real BMI of the child $\alpha\left(\mathrm{BMI}^{\alpha}\right.$ can be different from basicBMI, and it is the same principle we used for modeling MVPA ${ }^{\alpha}$ and basicMVPA). To simplify our modeling, we suppose that the corpulence is only influenced by: the age, the gender (via basicBMI), and the PA. In other words, we assume that, for $\alpha \in \mathrm{F}^{\mathrm{Child}}$, $\mathrm{BMI}^{\alpha}$ is basicBMI $\left(\mathrm{f}^{\mathrm{Age}}, \mathrm{f}^{\text {Gender }}\right)$ altered by the effect of the excess/lack of PA, i.e. MVPA ${ }^{\alpha}$. And we suppose that such influence is updated on a yearly basis, $\left\langle\mathrm{MVPA}^{\alpha}\right\rangle^{\mathrm{year}^{-1}}$.

In order to quantify 'How much should we increase/decrease the BMI for a given amount of minutes of MVPA?', we aggregated results offered by the literature (i.e. (Khan et al., 2014; Li et al., 2010)) and we calculated the effect of one daily minute of MVPA on the BMI of children between 6 and 18 years old. Let $\varepsilon^{\mathrm{MVPA} / \mathrm{BMI}} \in[-1 ; 1]$ be the coefficient resulting from this analysis (i.e. the quantified effect that we aggregated from the literature), specified by age, gender, and weight status. An example of how it was calculated is presented in Appendix A.

We assume that, considering all other factors stable, a normally active child has a normal weight status. That is to say, if a given child's MVPA over the last year is active, i.e.

$\left\langle\mathrm{MVPA}^{\alpha}\right\rangle^{\text {year }^{-1}}=$ basicMVPA$\left(\mathrm{f}^{\mathrm{Age}}, \mathrm{f}^{\mathrm{Gender}}\right)$, then the effect on $\mathrm{BMI}^{\alpha}$ is 0 . Otherwise, the excess $/$ lack of MVPA ${ }^{\alpha}$ influences $\mathrm{BMI}^{\alpha}$ depending on $\varepsilon^{\mathrm{MVPA} / \mathrm{BMI}}$ (itself depending on the age, gender, and current weight status). The excess/lack of MVPA during the last year can be calculated as: $\left\langle M_{M P A}^{\alpha}-\right.$ basicMVPA(f $\left.\left.{ }^{A g e}, f^{G e n d e r}\right)\right\rangle^{y^{e a r}{ }^{-1}}$. And by multiplying it by $\varepsilon^{\text {MVPA/BMI }}$, we obtain its total effect on the child's BMI. The total child's BMI though, BMI ${ }^{\alpha}$, also takes into account the natural growth of his/her corpulence basicBMI ${ }^{\alpha}$, which is updated based on the WHO references (WHO, 2007). The overall expression of how the child's corpulence evolves is stated in (5). The factor $\mathrm{f}^{\text {Corpulence }}$ is represented by the history of $\mathrm{BMI}^{\alpha}$ over the years.

$\mathrm{BMI}^{\alpha}=\mathrm{BMI}^{\alpha}+\varepsilon^{\mathrm{MVPA} / \mathrm{BMI}} *\left\langle\mathrm{MVPA}^{\alpha}-\operatorname{basicMVPA}\left(\mathrm{f}^{\mathrm{Age}}, \mathrm{f}^{\text {Gender }}\right)\right\rangle^{\text {year }^{-1}}$

Finally, we declare the update of the corpulence $\psi_{\mathrm{f}_{\mathrm{C}} \text {, }_{\mathrm{f}} \text { Gulence }}^{\mathrm{f}^{\mathrm{A}}{ }^{\mathrm{PA}}}$ that takes care of calculating the previous expression. This update is internal to the child; it includes only the child himself/herself, and by consequence inherits from $\psi_{\varnothing}^{\mathrm{F}^{\text {Individual }}}$.

iii. Summary of the risk factors and updates included in SimNCD ${ }^{\mathrm{ChO}}$

In this case of study, we included the risk factors $\left\{\mathrm{f}^{\mathrm{opp}}, \mathrm{f}^{\mathrm{Age}}, \mathrm{f}^{\text {Gender }}, \mathrm{f}^{\text {Attitude }}, \mathrm{f}^{\mathrm{PA}}, \mathrm{f}^{\text {Corpulence }}\right\}$ and the updates: $\mathrm{I}=\left\{\mathrm{I}^{\text {ChooseGoalActivity }}, \mathrm{I}^{\text {Move }}, \mathrm{I}^{\text {Engage }}, \mathrm{I}^{\text {Quit }}\right\} \cup$ 


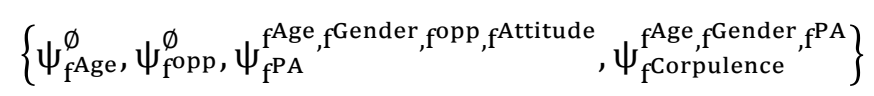

We summarize the considered risk factors and the relationships between them in Figure 3. The said relationships are represented by arrows, and they are applied in our ABM via the declared updates.

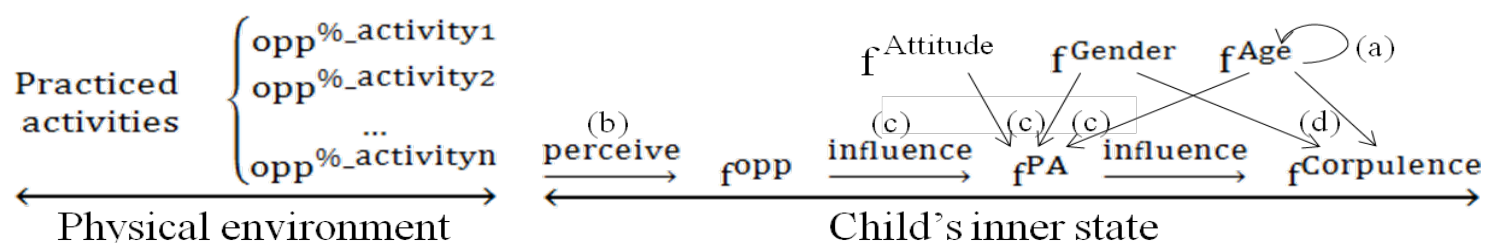

Figure 3. The risk factors included in $\operatorname{SimNCD}^{\mathrm{ChO}}$, with: (a) update the age: $\psi_{\mathrm{f}}^{\varnothing}$, (b) update the opportunity to

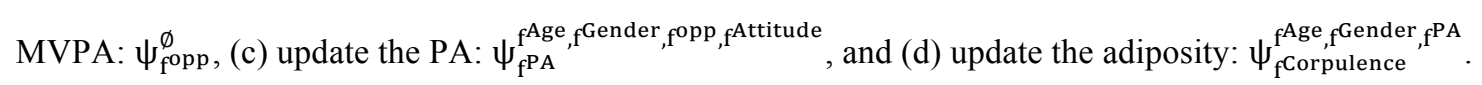

\subsubsection{Interactions}

The described updates of the risk factors occur in the behavior 'Update inner status' in the individual's behavioral model (Figure 2). We inject these updates in said model as shown in Figure 4, and in the ABM's interaction matrix as illustrated in Table 4. In the latter, we attribute priorities the same way we did for the generic interaction matrix (Table 1) by following the chronological order of the interactions. We would like to note that this case study does not include any communications between the individuals nor their mental attitudes. A further study should focus on these aspects.

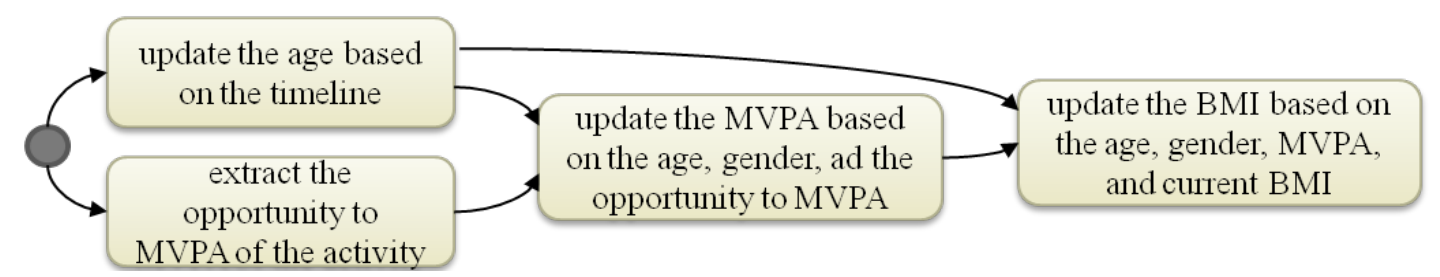

Figure 4. Specification of the behavior 'Update inner status' in the behavioral process in Figure 2.

Table 4. Modeling the influence of the physical environment on children's corpulence via their PA behavior: A specification of the generic matrix in Table 1 applied for $\operatorname{SimNCD}^{\mathrm{ChO}}$.

\begin{tabular}{|c|c|c|c|c|}
\hline $\begin{array}{l}\text { Target } \\
\text { Source }\end{array}$ & & $\varnothing$ & $F^{\text {Child }}$ & $\mathbf{F}^{\text {Avtivity }}$ \\
\hline \multirow[b]{2}{*}{$F^{\text {Child }}$} & \multirow[b]{2}{*}{ 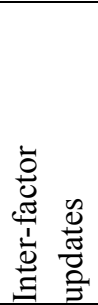 } & $\begin{array}{l}\left(\mathrm{I}_{\text {ChooseGoalActivity }}, 0\right) \\
\left(\mathrm{I}_{\text {Move }}, 1\right)\end{array}$ & - & $\begin{array}{l}\left(\mathrm{I}_{\text {Engage }}, 0,2\right) \\
\left(\mathrm{I}_{\text {Quit }}, 0,7\right)\end{array}$ \\
\hline & & 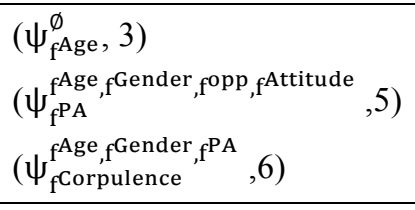 & - & $\left(\Psi_{\mathrm{fopp}}^{\emptyset}, 0,4\right)$ \\
\hline $\mathbf{F}^{\text {Activity }}$ & & - & - & - \\
\hline
\end{tabular}

\section{Results and discussion}

We have implemented SimNCD ${ }^{\mathrm{ChO}}$ using the framework JEDI (Kubera et al., 2011). We proceed with 
testing the simulator and comparing its results to the empirical data available in the literature. Then, we use the tool to predict the development of children's corpulence in different scenarios. In both the validation and the prediction, we first describe the model's calibration, simulate, and then interpret the results in the discussion section.

\subsection{Model calibration and validation}

In this section, we evaluate whether $\operatorname{SimNCD}{ }^{\mathrm{ChO}}$ can actually capture relevant aspects of the development of real children, and we verify if it can be used for future prediction tasks. In fact, it is very challenging to find detailed data to be fed to such a detailed modeling because of two main reasons. First, the available field studies have different contexts and hypothesis. They rarely include the same set of risk factors, and if so, different ways are often used to measure them. Second, most of these field studies do not follow the development of the individuals for long time spans. Hence, even though our modeling allows producing data about the PA and corpulence developments for long durations (e.g. from 6 to 18 years old), we believe it would be easier to validate it in separate age intervals. For that, we attempt in this section to reproduce field studies from the literature and compare their empirical data against the results of our simulations in order to validate the modeling for children from 8 to 13 years old. In each simulation, we create a population of 1000 children that mimic as closely as possible the population described in the field study that we want to simulate. We chose a population that is quite superior in number from the investigated field studies (1000 children) in order to mitigate the random choices at the initialization, and render the results more robust and trustworthy. The studies we included are interventions that focus on PA as an exposure factor and measure the consequent effect on children's corpulence. In order to assess their effectiveness, these studies compare between an intervention group that receives the program being tested, and a controlled group that receives a conventional treatment. In this evaluation, we simulated three interventions that replicate the works (Khan et al., 2014; Li et al., 2014; Peralta et al., 2009).

\subsubsection{Simulation of the intervention of Khan et al.}

The PA intervention (Khan et al., 2014) aimed to investigate the effects of PA on children's adiposity and cardio respiratory fitness. It consisted of $70 \mathrm{~min} /$ day of MVPA, 5 days/week for 9 months. It included 8 to 9 years old children ( $46 \%$ girls) that were assigned randomly to either the control group or the intervention group. In Table 5, we detail how we used the data provided by the field study (Khan et al., 2014) as calibration to $\mathrm{SimNCD}^{\mathrm{ChO}}$.

Table 5. Baseline data of the intervention (Khan et al., 2014) for both the control and intervention groups, and the corresponding calibration of $\mathrm{SimNCD}^{\mathrm{ChO}}$ to reproduce a simulation of the intervention.

\begin{tabular}{|c|c|c|c|}
\hline & & Data extracted from the article & Configuration of SimNCD ${ }^{\text {ChO }}$ \\
\hline \multirow{3}{*}{ 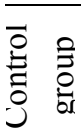 } & Age & $8.8 \pm 0.6$ years old & $8.8 \pm 0.6$ years old \\
\hline & Number & 110 child (49 girls) & $1000(445 \text { girls })^{\mathrm{a}}$ \\
\hline & BMI & $62 \%$ underweight or normal, with & $62 \%$ have BMI: $16.3 \pm 1.5^{\mathrm{b}}$ \\
\hline
\end{tabular}




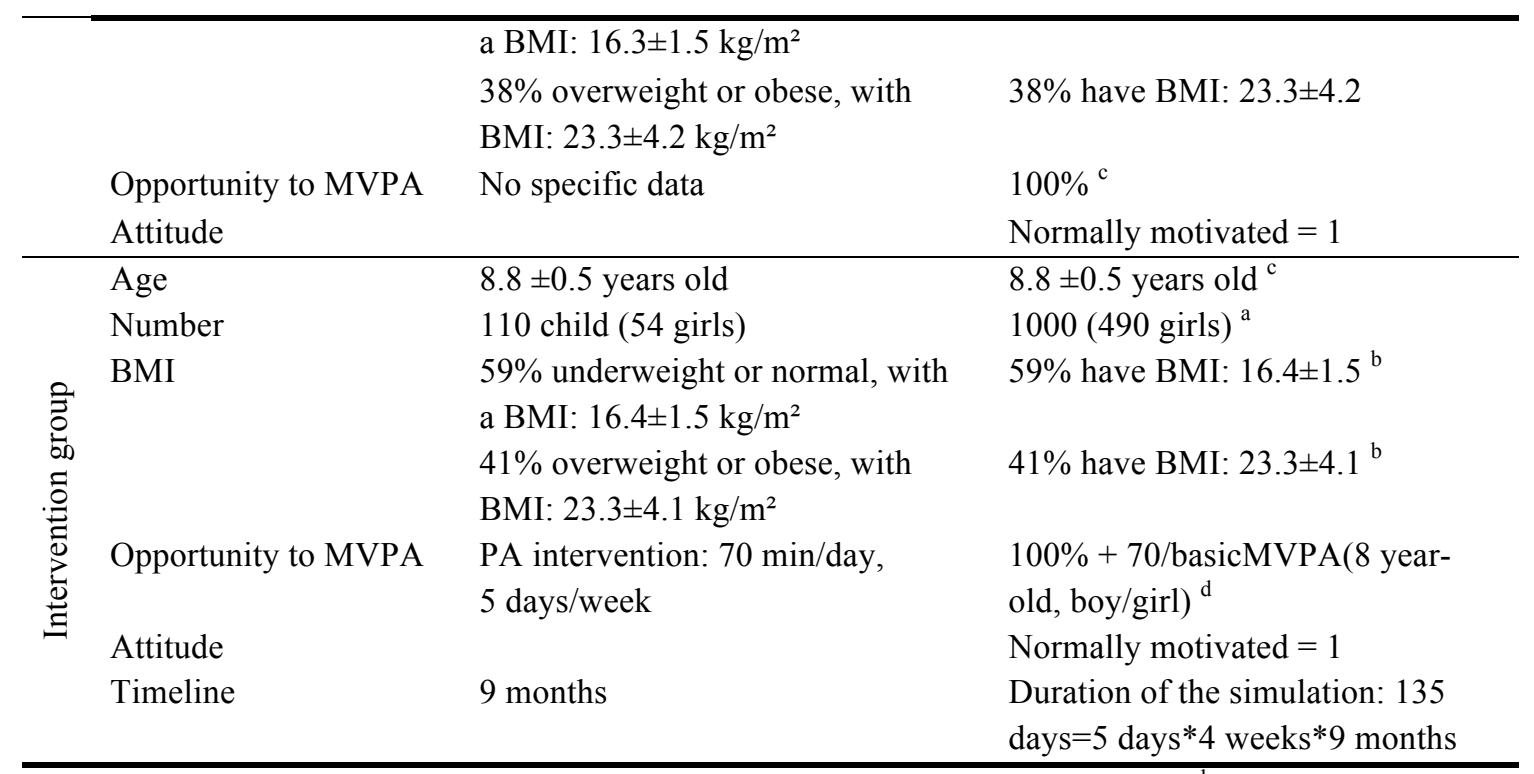

${ }^{\mathrm{a}}$ The Number of boys or girls corresponds to the percentage in the real population, ${ }^{\mathrm{b}}$ Set randomly, ${ }^{\mathrm{c}}$ The total opportunity to MVPA of the daily practiced activities is $100 \%$, e.g. go to school then play in the garden, ${ }^{\mathrm{d}}$ We add an activity that offers an opportunity to MVPA equivalent to $70 \mathrm{~min}$, based on basicMVPA(8 year-old, boy/girl).

We ran the simulations of both groups and we registered the children's BMI at the end of the ninemonth intervention period. We calculated the mean BMI of each group and compared them to the corresponding field data. The mean, max, and min BMI are presented in Figure 5. We notice that the results are close to the empirical data; the minimal difference in average BMI between the $\mathrm{SimNCD}^{\mathrm{ChO}}$ simulations and the real data from (Khan et al., 2014) is $0.27 \mathrm{~kg} / \mathrm{m}^{2}$ for underweight and normal children in the control group, whereas the maximum difference $0.7 \mathrm{~kg} / \mathrm{m}^{2}$ was found in the overweight and obese children in the intervention group.

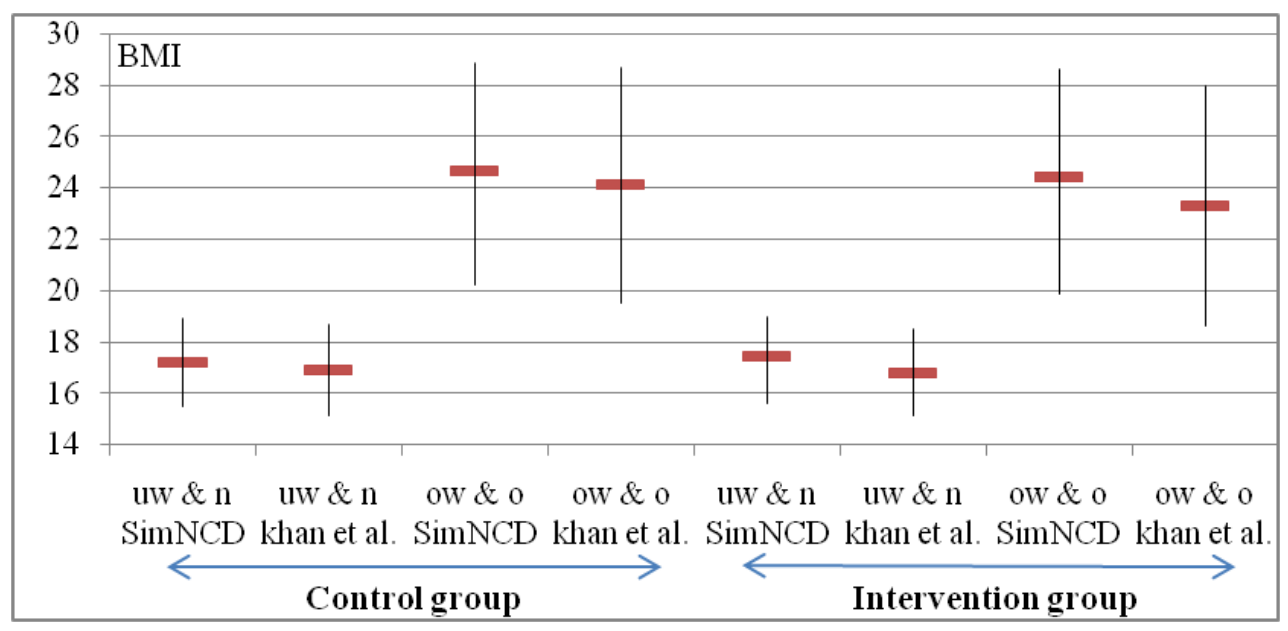

Figure 5. Comparison between the intervention (Khan et al., 2014) and its reproduction using $\operatorname{SimNCD}^{\mathrm{ChO}}$. The results are specified by adiposity (uw \& n: underweight and normal, ow \& o: overweight and obese).

\subsubsection{Simulation of the intervention of Perlata et al.}

Perlata et al. carried out a six months intervention on 12 to 13 years old boys in Sydney (Peralta et al., 2009). The participants were randomly assigned to either the control or the intervention group, and the 
latter participated in extra PA sessions. Following the example of the first study, we extracted the key data to calibrate $\mathrm{SimNCD}{ }^{\mathrm{ChO}}$. In this one, however, the MVPA data were stated in more details, e.g. boys from the intervention group spent $123.3 \pm 62 \mathrm{~min} /$ day of MVPA. To include such information in our simulation, we modeled the mean, i.e. $123 \mathrm{~min} /$ day, as the daily available opportunity to MVPA, and each child had an attitude in an interval equivalent to $[-62 ;+62]$, calculated based on basicMVPA. This way, the children can gather different amounts of MVPA while staying within the range specified by the study. The simulation consisted of 1000 boys that corresponded to the mentioned extracted data. The results (in Figure 6(a)) led to a mean BMI of $0.2 \%$ higher in the control group and $2.5 \%$ higher in the intervention group when compared to the empirical data.

\subsubsection{Simulation of the intervention of Li et al.}

The work of Li et al. is based on a 12 week intervention that included children from 9 to 13 years old, with a prevalence of $46.5 \%$ girls in the control group and $47.4 \%$ girls in the intervention group (Li et al., 2014). We used the data of the study as calibration parameters to SimNCD ${ }^{\mathrm{ChO}}$. Among other parameters, we set the BMI distribution and the prevalence of genders. The comparative results are presented in Figure 6(b), and it shows very close results between the means of BMI at the end of the intervention, with a $-0.32 \mathrm{~kg} / \mathrm{m}^{2}$ gap of the mean BMI for the control group, and $0.09 \mathrm{~kg} / \mathrm{m}^{2}$ gap in the case of the intervention group.

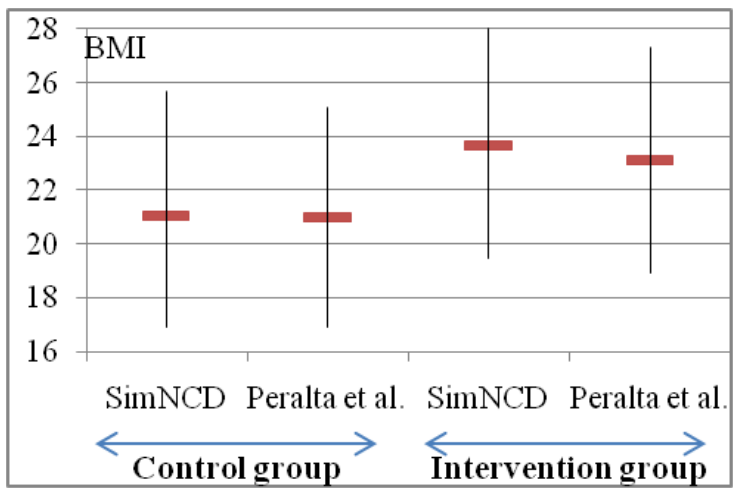

(a)

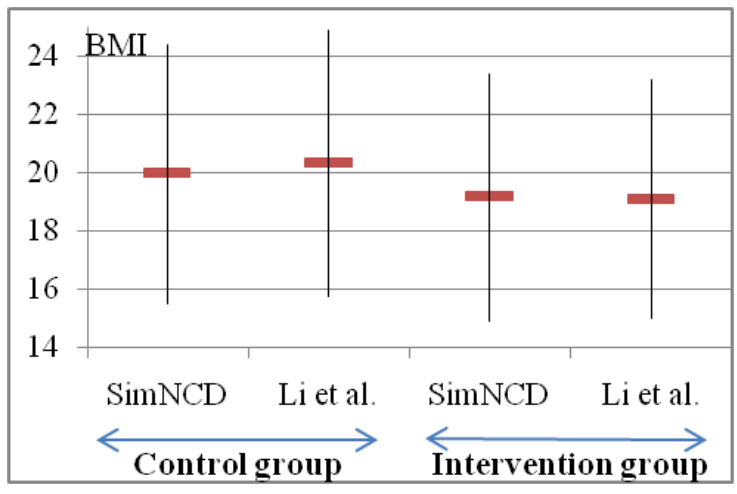

(b)

Figure 6. Comparison between the data on the interventions (Peralta et al., 2009) in figure (a) and (Li et al., 2014 ) in figure (b), and their replication using $\operatorname{SimNCD}^{\mathrm{ChO}}$. The follow-up results are presented for both the control and the intervention groups of each intervention.

\subsubsection{Discussion}

SimNCD ${ }^{\mathrm{ChO}}$ allowed a detailed simulation of children's daily activities and the influence of the latter on the PA levels as well as the corpulence variations. The virtual reproduction of the three studies (Khan et al., 2014; Li et al., 2014; Peralta et al., 2009) provided results that were close to the empirical data. Besides that, the simulated children and the real ones both had the same distribution of their corpulence statuses based on the WHO references. These results prove that $\mathrm{SimNCD}^{\mathrm{ChO}}$ is useful for simulating real life scenarios and interventions. One could, for example, add different activities and 
analyze their impact on the children's health.

In fact, the empirical data, in the literature about the relationship between the PA and the corpulence development for children, can be very different and even contradictory at times. Such divergence is due to many reasons like the different hypothesis that these studies rely on, the differences in the techniques used to measure the considered risk factors, and the diversity of the many other factors (e.g. economical and social) in the contexts in which the studies were undertaken (even if these factors were not analyzed and brought out in the study itself). These disparities can be mirrored in the results and the interpretations done by the health experts. We surpassed this difficulty by calculating a coefficient that encloses several studies, i.e. the coefficient $\varepsilon^{\mathrm{MVPA} / \mathrm{BMI}}$ that was aggregated from the literature. Moreover, further investigation should be done in order to validate the proposed modeling for all the ages between 6 and 18 years old. Since it is very difficult to find time-series data for individual children over long time spans, a validation could be performed separately by age intervals. Withal, the main goal of this paper is to present a formalism, SimNCD, which can be specified in the study of NCDs. And by providing a proof of concept, $\operatorname{SimNCD}^{\mathrm{ChO}}$ that approached results of empirical data, this confirms the usability of SimNCD. However, we do not claim that SimNCD is 'the' solution for modeling and simulating all NCDs' dynamics. Some specific areas of study could fall beyond the reach of our modeling, such as the genetic aspect of the NCDs or the studies in molecular biology. SimNCD is a candidate tool for modeling NCD dynamics that are based on the individuals' behaviors, their communications with each other and with their physical environment. Unlike formal models, SimNCD does not restrict the dynamics of the system, such as the use of stochastic dynamics in SIENA (Snijders et al., 2010). On the other hand, SIENA offers a set of already modeled micromechanisms that translate some real life network tendencies, like reciprocity or homophily (choice of network ties based on similarity of relevant attributes). Our novice tool, SimNCD, is yet to develop ready-to-use mechanisms that can help public health researchers understand the use of SimNCD. It will also simplify and quicken their design of their own models, and allow them to widen the scope of their studies. For that, a more thorough study of the mechanisms that govern inter-factor and interindividual relationships in different NCDs will be needed.

\subsection{Simulation for prediction}

$\operatorname{SimNCD}^{\mathrm{ChO}}$ allows the simulation of different scenarios in order to obtain insights about real children and study the effect of different behaviors and PA programs on the children's BMI during their growth. In this paper, the simulations are based on five main factors: the age, the gender, the daily opportunity to MVPA offered by the physical environment (unfavorable, favorable, or highly favorable), the child's attitude (unmotivated, motivated, or highly motivated), and the initial corpulence status (normal, overweight, or obese).

\subsubsection{Model calibration}


We depicted three scenarios that we describe in Table 6. Each scenario was simulated for both genders with a population of 1000 children. All children were started at 6 years old and the physical environment was set to 'highly favorable'. We note that, the BMI was set according to the desired corpulence status, yet at the same time not exactly equal to the WHO recommendations (WHO, 2007). In fact, these latter represent the cutoff points for the BMI statuses, and when used to initialize the children's BMI, any slight decrease in the BMI would immediately result in a change of the corpulence status. Thus, the children were given BMIs equal to basicBMI(6 year-old, boy/girl) +1 . With these scenarios, we attempt to study the effect of a highly favorable physical environment on the evolution of the BMI in the case of overweight and obese children.

Table 6. Calibration of $\mathrm{SimNCD}^{\mathrm{ChO}}$ for the simulation of scenarios that aim to predict children's corpulence variations in a highly favorable physical environment.

\begin{tabular}{|c|c|c|c|c|}
\hline \multicolumn{2}{|c|}{ Simulation parameters } & Scenario 1 & Scenario 2 & Scenario 3 \\
\hline \multirow{5}{*}{ 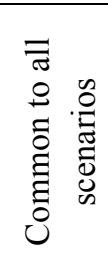 } & Age & \multicolumn{3}{|l|}{6 years old } \\
\hline & Number & \multicolumn{3}{|l|}{1000 of each gender } \\
\hline & Opportunity to & \multirow{2}{*}{\multicolumn{3}{|c|}{$\begin{array}{l}\text { Highly favorable physical environment (i.e. }>100 \% \text { ): set to } 130 \% \text {. In other } \\
\text { words, the sum of the opportunities of the practiced activities gathered } \\
\text { daily by the children is } 130 \% \text {. }\end{array}$}} \\
\hline & MVPA & & & \\
\hline & Timeline & \multicolumn{3}{|c|}{13 years: 1 year is equal to 180 school days } \\
\hline \multirow{5}{*}{ 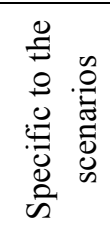 } & $\mathrm{BMI}^{\mathrm{a}}$ & Overweight & Obese & Obese \\
\hline & & boys: $18.11 \mathrm{~kg} / \mathrm{m}^{2}$ & boys: $19.76 \mathrm{~kg} / \mathrm{m}^{2}$ & boys: $19.76 \mathrm{~kg} / \mathrm{m}^{2}$ \\
\hline & & girls: $18.37 \mathrm{~kg} / \mathrm{m}^{2}$ & girls: $20.45 \mathrm{~kg} / \mathrm{m}^{2}$ & girls: $20.45 \mathrm{~kg} / \mathrm{m}^{2}$ \\
\hline & Attitude ${ }^{b}$ & Unmotivated: & Unmotivated: & Highly motivated: \\
\hline & & $60 \% \pm 10 \%$ & $60 \% \pm 10 \%$ & $140 \% \pm 10 \%$ \\
\hline
\end{tabular}

${ }^{\mathrm{a}} \mathrm{BMI}=$ basicBMI $(6$ year-old, boy/girl $)+1,{ }^{\mathrm{b}}$ a random value in $[-10 \% ;+10 \%]$ is added to the Attitude.

\subsubsection{Predictive results and discussion}

We present the simulation results in Table 7 and Figure 7. In Figure 7, we draw the simulation results with some WHO figures (WHO, 2007). These levels do not show the development of children based on the activity level. They rather represent the levels of BMI that count as normal, overweight, and obese. The WHO references are only used to show how the corpulence statuses of the simulated populations change over time. Compared to those references, our results (Figure 7) could be considered as plausible. They also confirm the strong influence of the PA on the children's adiposity (Guinhouya, 2012; Katzmarzyk et al., 2015).

Table 7. Results of the simulation of three scenarios occurring in a highly favorable physical environment. Each column represents the variations of the mean BMI of 1000 children: The children are overweight and unmotivated in scenario 1, obese and unmotivated in scenario 2 , obese and highly motivated in scenario 3 . The mean BMI is based on the WHO references (WHO, 2007): green or ' $\mathrm{n}$ ' = normal, orange or 'ow' = overweight, red or 'o' = obese.

Scenario 1:

overweight - unmotivated
Scenario 2:

obese - unmotivated
Scenario 3:

obese - highly motivated 


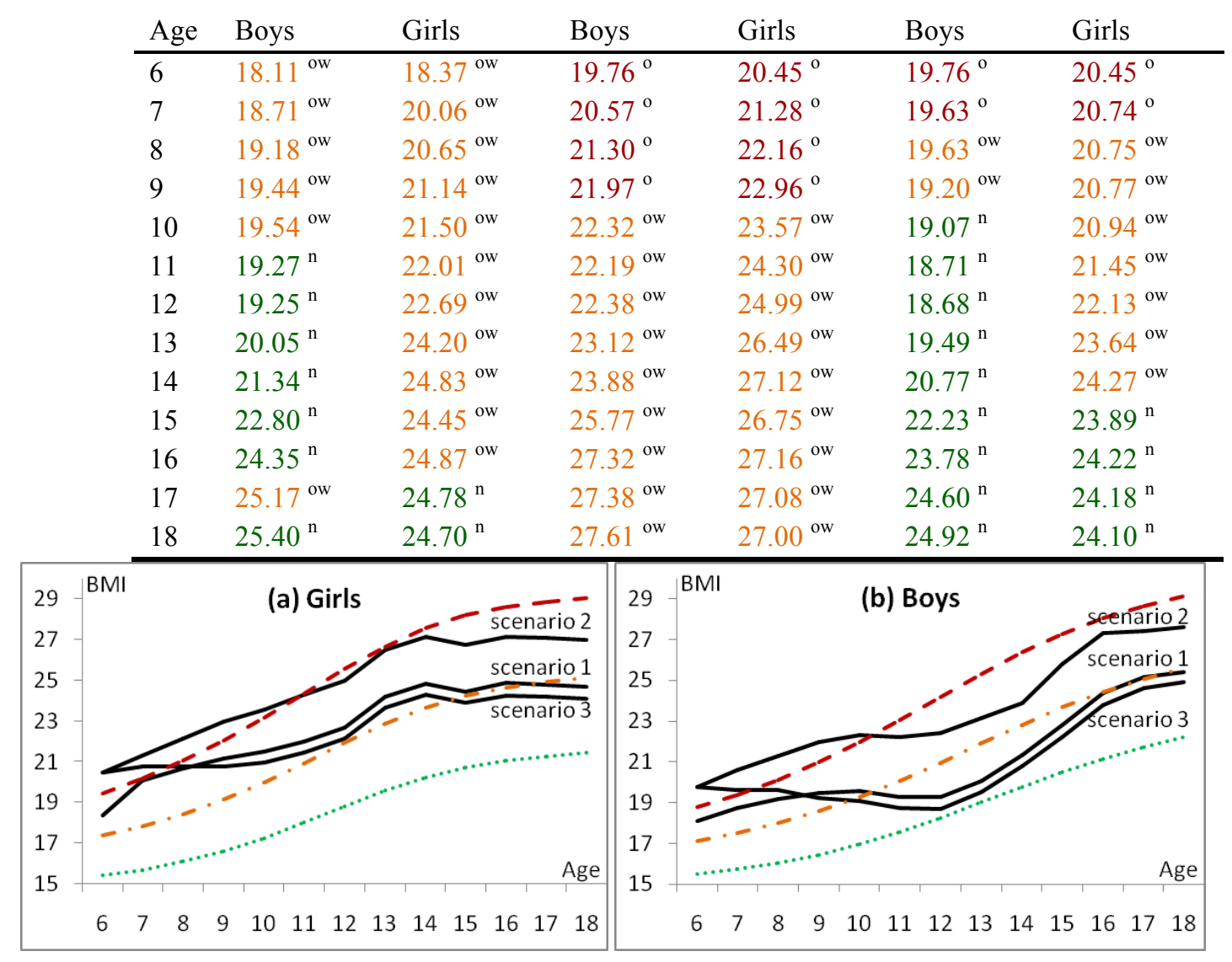

Figure 7. Results of the simulation of the three scenarios presented in Table 6. The solid lines represent the three scenarios simulated for girls/boys, and the other lines represent the BMI WHO references (WHO, 2007): dotted $=$ normal, dash-dot $=$ overweight, dashed $=$ obese.

In addition, in all scenarios we notice that a highly favorable physical environment is beneficial for the BMI, independently of the initial corpulence status and PA level. This interpretation emphasizes the importance of the physical environment in the prevention of childhood obesity, a fact that is supported by many studies in the literature (Epstein et al., 2012; Grafova, 2008; WHO, 2014).

In the first scenario, the boys' corpulence status improved at all ages except 17, where it changed from normal to overweight despite being in a highly favorable physical environment. This suggests that, even when subjected to the same conditions, the child's corpulence does not necessarily respond the same way at all ages. This would lead us to focus on PA programs targeting specific age segments. We also notice that the first and third scenarios were very close after the age of 8 when we compare children of the same gender; the difference of mean BMI $\leq 0.6 \mathrm{~kg} / \mathrm{m}^{2}$ at all ages after 8 . In the first scenario, children were unmotivated and overweight, while in the third they were highly motivated but initially obese. This could suggest that a positive PA behavior can compensate for a less favorable initial BMI.

The second scenario depicted obese and unmotivated children. The BMI improved for both genders despite their disadvantageous initial states. This points to the direction of the importance of the physical environment. Nevertheless, the latter was not enough to lead the children to normal adiposity. 
We could suppose that other efforts need to be combined with the favorable environment in order to lead the children safely back from harmful states of corpulence.

Besides that, overweight and obesity seem more persistent with girls at all ages and all scenarios. This confirms the difference in the dynamics of BMI as a function of gender (WHO, 2007), and it might suggest that it is more difficult to prevent obesity in girls. Ness et al. stipulate the same in a crosssectional analysis (Ness et al., 2007), where they concluded that the PA had an inverse association with obesity, and that this association was stronger in boys. Consequently, programs developed towards girls probably need to be long enough to be successful on their BMI curve.

\section{Conclusions}

In this paper, we proposed SimNCD, a generic interaction-oriented ABM for the study of NCDs. Basically, the system aims to model individuals living within a social and a physical environment. The individuals engage in activities in a daily manner, and these activities influence their development. By consequence, they steer the individual's health vulnerability to acquire or maintain the NCDs. We presented a formal description of SimNCD based on IODA (Kubera et al., 2011), and we focused on the definition of the risk factors and the updates that control their development. The proposed ABM helps predict for a better decision-making by simulating realistic scenarios in order to study the impact of different health programs on the development of the corpulence of children. Such virtual simulation obviates the temporal dimension by simulating long periods in a matter of seconds, and helps avoid the costs and effects of such field studies conducted in real life.

As a proof of concept, we specified SimNCD and modeled SimNCD ${ }^{\mathrm{ChO}}$; an ABM for the study of the complex relationships between the PA behaviors and children's corpulence variations. To limit our scope, we focused on modeling the influence of the physical environment, i.e. the opportunities to MVPA, on children's adiposity. SimNCD ${ }^{\mathrm{ChO}}$ adheres to the complexity of the PA behaviors of children, and aims to model their mechanisms. It is based on the statistic studies and the existing epidemiological literature, including references (Guinhouya, 2012; WHO, 2007), recommendations (WHO, 2014) and field studies (Li et al., 2010). To validate the modeling, we successfully reproduced empirical data from several PA field interventions (Khan et al., 2014; Li et al., 2014; Peralta et al., 2009). While such evaluation does not evaluate the system for all ages from 6 to 18 years old, it proves the validity of the basic structure of SimNCD and SimNCD ${ }^{\mathrm{ChO}}$, and that the proposed design indeed allows a testing of real life behavioral interventions.

As future work, SimNCD could be extended to include cognitive reasoning mechanisms that allow the individuals to have mental attitudes, like preferences. Besides that, a focus on modeling generic interindividual communications, e.g. peer influence, could improve and widen the range of possible implementations of the proposed formalism. This could call for modeling specific kinds of agents, such as the scheduler agent (North, 2014). SimNCD is aimed to serve as a tool in public health policies for the study of NCDs where the agent's behavior greatly influences the factors predisposing 
to that disease. It would therefore be interesting to produce versions of SimNCD that focus the study of different NCDs, like SimNCD ${ }^{\mathrm{ChO}}$.

\section{A Appendix.}

In this appendix, we present an example of how we quantified the effect of one daily minute of MVPA on the child's BMI. Our calculations were based on results offered by field studies with objectively measured MVPA (in minutes) and BMI (in $\mathrm{kg} / \mathrm{m}^{2}$ ). In this paper, we used the aggregated effects of results from the literature as a coefficient $\varepsilon^{\mathrm{MVPA} / \mathrm{BMI}}$, and the outcome calculated from one study $\mathrm{x}$ as $\varepsilon_{\mathrm{x}}^{\mathrm{MVPA} / \mathrm{BMI}}$. Thus, the aggregated coefficient $\varepsilon^{\mathrm{MVPA} / \mathrm{BMI}}$ was calculated as the mean of the different coefficients $\varepsilon_{\mathrm{x}}^{\mathrm{MVPA} / \mathrm{BMI}}$. In this example, we consider the work by Li et al. (Li et al., 2010) and we calculate $\varepsilon_{(\mathrm{Li} \text { et al.) }}^{\mathrm{MVPA} \text {. }}$. Li et al. performed a PA intervention on 4700 students, aged between 8 to 11 years at baseline. This intervention consisted of a daily 20 min of MVPA during a year. One of the most important outcomes from the study by $\mathrm{Li}$ et al. was the computation of the difference in BMI (post-intervention - baseline) at the end of the intervention, according to gender: the mean BMI effect was $-0.17 \mathrm{~kg} / \mathrm{m}^{2}$ for girls, and $-0.15 \mathrm{~kg} / \mathrm{m}^{2}$ for boys. We calculate the coefficient $\varepsilon_{(\mathrm{Li} \mathrm{et} \mathrm{al.)}}^{\mathrm{MVA} \text { ) }}$ as: $\varepsilon_{(\text {Li et al. })}^{\mathrm{MVPA} / \mathrm{BMI}}=$ BMI change $/$ MVPA duration $=\left\{\begin{array}{l}-0.17 / 20 \text { (girls) } \\ -0.15 / 20 \text { (boys) }\end{array}=\left\{\begin{array}{l}-0,0085 \text { (girls) } \\ -0,0075 \text { (boys) }\end{array}\right.\right.$

\section{References}

Auchincloss, A.H., Riolo, R.L., Brown, D.G., Cook, J., Diez Roux, A. V., 2011. An agent-based model of income inequalities in diet in the context of residential segregation. Am. J. Prev. Med. 40, 303-311. doi:10.1016/j.amepre.2010.10.033

Aziza, R., Borgi, A., Zgaya, H., Guinhouya, B., 2014. A Multi-agent Simulation: The Case of Physical Activity and Childhood Obesity, in: Distributed Computing and Artificial Intelligence, 11th International Conference SE - 42, Advances in Intelligent Systems and Computing. pp. 359-367. doi:10.1007/978-3319-07593-8_42

Bagdasaryan, A., 2011. Discrete dynamic simulation models and technique for complex control systems. Simul. Model. Pract. Theory 19, 1061-1087. doi:10.1016/j.simpat.2010.12.010

Behzadi, S., Alesheikh, A., 2013. Introducing a novel model of belief-desire-intention agent for urban land use planning. Eng. Appl. Artif. Intell. 26, 2028-2044. doi:10.1016/j.engappai.2013.06.015

Bonabeau, E., 2002. Agent-based modeling: methods and techniques for simulating human systems. Proc. Natl. Acad. Sci. U. S. A. 99, 7280-7287. doi:10.1073/pnas.082080899

Caballero, A., Botía, J., Gómez-Skarmeta, A., 2011. Using cognitive agents in social simulations. Eng. Appl. Artif. Intell. 24, 1098-1109. doi:10.1016/j.engappai.2011.06.006

Crooks, A.T., Hailegiorgis, A.B., 2014. An agent-based modeling approach applied to the spread of cholera. Environ. Model. Softw. 62, 164-177. doi:10.1016/j.envsoft.2014.08.027

Dobe, M., 2012. Health promotion for prevention and control of non-communicable diseases: unfinished agenda. Indian J. Public Health 56, 180-6. doi:10.4103/0019-557X.104199 
Epstein, L.H., Raja, S., Daniel, T.O., Paluch, R. a, Wilfley, D.E., Saelens, B.E., Roemmich, J.N., 2012. The built environment moderates effects of family-based childhood obesity treatment over 2 years. Ann. Behav. Med. 44, 248-58. doi:10.1007/s12160-012-9383-4

Garrison, L.A., Babcock, D.S., 2009. Alcohol consumption among college students: An agent-based computational simulation. Complexity 14, 35-44. doi:10.1002/cplx.20259

Grafova, I.B., 2008. Overweight children: Assessing the contribution of the built environment. Prev. Med. (Baltim). 47, 304-308. doi:10.1016/j.ypmed.2008.04.012

Guinhouya, B., 2012. Physical activity during the development of the child. Medecine Sciences Publications, Paris.

Guinhouya, B., 2010. Physical activity of schoolchildren in France. The paradox of a public health priority! Rev. Epidemiol. Sante Publique 58, 255-67. doi:10.1016/j.respe.2010.02.111

Guinhouya, B., Apété, G.K., Hubert, H., 2010. The determinants of habitual physical activity (HPA) in children: update and implications for care and prevention options in pediatric overweight/obesity. Rev. Epidemiol. Sante Publique 58, 49-58. doi:10.1016/j.respe.2009.10.007

Hammond, R. a, Ornstein, J.T., 2014. A model of social influence on body mass index. Ann. N. Y. Acad. Sci. 1331, 1-9. doi:10.1111/nyas.12344

Hussain, F., Ramanathan, A., Pullum, L., Jha, S., 2014. EpiSpec: A Formal Specification Language for Parameterized Agent-Based Models against Epidemiological Ground Truth. Eecs.Ucf.Edu.

Kantamneni, A., Brown, L.E., Parker, G., Weaver, W.W., 2015. Survey of multi-agent systems for microgrid control. Eng. Appl. Artif. Intell. 45, 192-203. doi:10.1016/j.engappai.2015.07.005

Katzmarzyk, P.T., Barreira, T. V., Broyles, S.T., Champagne, C.M., Chaput, J.-P., Fogelholm, M., Hu, G., Johnson, W.D., Kuriyan, R., Kurpad, A., Lambert, E. V., Maher, C., Maia, J., Matsudo, V., Olds, T., Onywera, V., Sarmiento, O.L., Standage, M., Tremblay, M.S., Tudor-Locke, C., Zhao, P., Church, T.S., 2015. Relationship between lifestyle behaviors and obesity in children ages 9-11: Results from a 12country study. Obesity 23, 1696-1702. doi:10.1002/oby.21152

Khan, N. a, Raine, L.B., Drollette, E.S., Scudder, M.R., Pontifex, M.B., Castelli, D.M., Donovan, S.M., Evans, E.M., Hillman, C.H., 2014. Impact of the FITKids physical activity intervention on adiposity in prepubertal children. Pediatrics 133, e875-83. doi:10.1542/peds.2013-2246

Kubera, Y., Mathieu, P., Picault, S., 2011. IODA: an interaction-oriented approach for multi-agent based simulations. Auton. Agent. Multi. Agent. Syst. 23, 303-343. doi:10.1007/s10458-010-9164-Z

Li, X., Lin, S., Guo, H., Huang, Y., Wu, L., Zhang, Z., Ma, J., Wang, H., 2014. Effectiveness of a school-based physical activity intervention on obesity in school children : a nonrandomized controlled trial. BMC Public Health 14, 1-12. doi:10.1186/1471-2458-14-1282

Li, Y.P., Hu, X.Q., Schouten, E.G., Liu, A.L., Du, S.M., Li, L.Z., Cui, Z.H., Wang, D., Kok, F.J., Hu, F.B., Ma, G.S., 2010. Report on childhood obesity in China (8): Effects and sustainability of physical activity intervention on body composition of Chinese youth. Biomed. Environ. Sci. 23, 180-187. doi:10.1016/S0895-3988(10)60050-5

Mei, S., Zarrabi, N., Lees, M., Sloot, P.M.A., 2015. Complex agent networks: An emerging approach for modeling complex systems. Appl. Soft Comput. 37, 311-321. doi:10.1016/j.asoc.2015.08.010

Ness, A.R., Leary, S.D., Mattocks, C., Blair, S.N., Reilly, J.J., Wells, J., Ingle, S., Tilling, K., Smith, G.D., 
Riddoch, C., 2007. Objectively measured physical activity and fat mass in a large cohort of children. PLoS Med. 4, e97. doi:10.1371/journal.pmed.0040097

Nianogo, R. a., Arah, O. a., 2015. Agent-Based Modeling of Noncommunicable Diseases: A Systematic Review.

Am. J. Public Health 105, e20-e31. doi:10.2105/AJPH.2014.302426

Nicolet, J.L., 2010. Risks and complexity. Harmattan Editions.

North, M.J., 2014. A theoretical formalism for analyzing agent-based models. Complex Adapt. Syst. Model. $2,3$. doi:10.1186/2194-3206-2-3

Orr, M.G., Galea, S., Riddle, M., Kaplan, G.A., 2014. Reducing racial disparities in obesity: Simulating the effects of improved education and social network influence on diet behavior. Ann. Epidemiol. 24, 563-569. doi:10.1016/j.annepidem.2014.05.012

Padgham, L., Winikoff, M., 2005. Developing Intelligent Agent Systems: A Practical Guide, Wiley Series in Agent Technology. Wiley.

Peralta, L.R., Jones, R. a, Okely, A.D., 2009. Promoting healthy lifestyles among adolescent boys: the Fitness Improvement and Lifestyle Awareness Program RCT. Prev. Med. (Baltim). 48, 537-42. doi:10.1016/j.ypmed.2009.04.007

Pogson, M., Smallwood, R., Qwarnstrom, E., Holcombe, M., 2006. Formal agent-based modelling of intracellular chemical interactions. Biosystems 85, 37-45. doi:10.1016/j.biosystems.2006.02.004

Reiner, R.C., Stoddard, S.T., Scott, T.W., 2014. Socially structured human movement shapes dengue transmission despite the diffusive effect of mosquito dispersal. Epidemics 6, 30-36. doi:10.1016/j.epidem.2013.12.003

Russell, S.J., Norvig, P., 2013. Artificial Intelligence: A Modern Approach, Always learning. Pearson.

Schaefer, D.R., Adams, J., Haas, S.A., 2013. Social Networks and Smoking: Exploring the Effects of Peer Influence and Smoker Popularity Through Simulations. Heal. Educ. Behav. 40, 24S-32S. doi:10.1177/1090198113493091

Segovia-Juarez, J.L., Ganguli, S., Kirschner, D., 2004. Identifying control mechanisms of granuloma formation during M. tuberculosis infection using an agent-based model. J. Theor. Biol. 231, 357-376. doi:10.1016/j.jtbi.2004.06.031

Shoham, D. a, Tong, L., Lamberson, P.J., Auchincloss, A.H., Zhang, J., Dugas, L., Kaufman, J.S., Cooper, R.S., Luke, A., 2012. An actor-based model of social network influence on adolescent body size, screen time, and playing sports. PLoS One 7, e39795. doi:10.1371/journal.pone.0039795

Siddiqa, A., Niazi, M., 2013. A Novel Formal Agent-Based Simulation Modeling Framework of an AIDS Complex Adaptive System. Int. J. Agent Technol. Syst. 5, 33-53. doi:10.4018/ijats.2013070103

Snijders, T.A.B., van de Bunt, G.G., Steglich, C.E.G., 2010. Introduction to stochastic actor-based models for network dynamics. Soc. Networks 32, 44-60. doi:10.1016/j.socnet.2009.02.004

Wasik, S., Jackowiak, P., Figlerowicz, M., Blazewicz, J., 2014. Multi-agent model of hepatitis C virus infection. Artif. Intell. Med. 60, 123-131. doi:10.1016/j.artmed.2013.11.001

WHO, 2014. Global Status Report On Noncommunicable Diseases 2014.

WHO, 2007. New Child Growth Standards. World Heal. Organ. doi:10.1037/e569412006-008

Wooldridge, M., 2009. An Introduction to MultiAgent Systems. Wiley.

Yang, Y., Diez Roux, A. V., Auchincloss, A.H., Rodriguez, D.A., Brown, D.G., 2011. A spatial agent-based 
model for the simulation of adults' daily walking within a city. Am. J. Prev. Med. 40, 353-361. doi:10.1016/j.amepre.2010.11.017

Yang, Y., Diez-Roux, A., Evenson, K.R., Colabianchi, N., 2014. Examining the impact of the walking school bus with an agent-based model. Am. J. Public Health 104, 1196-203. doi:10.2105/AJPH.2014.301896

Zhang, D., Giabbanelli, P.J., Arah, O.A., Zimmerman, F.J., 2014. Impact of Different Policies on Unhealthy Dietary Behaviors in an Urban Adult Population: An Agent-Based Simulation Model. Am. J. Public Health 104, 1217-1222. doi:10.2105/AJPH.2014.301934

Zhang, J., Tong, L., Lamberson, P.J., Durazo-Arvizu, R.A., Luke, A., Shoham, D.A., 2015. Leveraging social influence to address overweight and obesity using agent-based models: The role of adolescent social networks. Soc. Sci. Med. 125, 203-213. doi:10.1016/j.socscimed.2014.05.049 